\title{
An Mtb-Human Protein-Protein Interaction Map Reveals that Bacterial LpqN Antagonizes CBL, a Host Ubiquitin Ligase that Regulates the Balance Between Anti-Viral and Anti- Bacterial Responses
}

Bennett H. Penn ${ }^{1}$, Zoe Netter ${ }^{1}$, Jeffrey R. Johnson ${ }^{2}$, John Von Dollen², Gwendolyn M. Jang ${ }^{2}$, Tasha Johnson ${ }^{2}$, Yamini M. Ohol ${ }^{3}$, Cyrus Maher ${ }^{4}$, Samantha L. Bell ${ }^{3}$, Kristina Geiger ${ }^{1}$, Xiaotang $\mathrm{Du}^{3}$, Alex Choi ${ }^{3}$, Trevor Parry ${ }^{3}$, Mayumi Naramura ${ }^{5}$, Chen Chen ${ }^{7}$, Stefanie Jaeger ${ }^{2}$, Michael Shales $^{2}$, Dan A. Portnoy ${ }^{7}$, Ryan Hernandez ${ }^{4}$, Laurent Coscoy ${ }^{1}$, Jeffery S. Cox ${ }^{1,8^{*}}$ and Nevan J. $\operatorname{Krogan}^{2,8,9^{*}}$

\section{Author Affiliations}

1. Department of Molecular and Cell Biology, University of California, Berkeley, CA 94720, USA.

2. Department of Cellular and Molecular Pharmacology, University of California, San Francisco, CA 94158, USA, QBI, Quantitative Biosciences Institute, University of California, San Francisco, CA 94148, USA; Gladstone Institutes, San Francisco, CA 94158, USA.

3. Department of Microbiology and Immunology, Program in Microbial Pathogenesis and Host Defense, University of California, San Francisco, CA 94158, USA.

4. Department of Epidemiology and Biostatistics, University of California, San Francisco, CA, USA.

5. Eppley Institute for Research in Cancer and Allied Diseases, University of Nebraska, Omaha, NE 68182, USA.

6. Department of Medicine, University of California, San Francisco, San Francisco, CA 94143, USA.

7. School of Public Health, University of California, Berkeley, Berkeley, CA 94720

8. These authors contributed equally to this work.

9. Lead Author.

* Correspondence: nevan.krogan@ucsf.edu (N.J.K.), jeff.cox@berkeley.edu (J.S.C).

\section{SUMMARY}

Although macrophages are armed with potent anti-bacterial functions, Mycobacterium tuberculosis $(\mathrm{Mtb})$ replicates inside these innate immune cells. Determinants of macrophageintrinsic bacterial control, and the Mtb strategies to overcome them are poorly understood. To further study these processes, we used a systematic affinity tag purification mass spectrometry (AP-MS) approach to identify 187 Mtb-human protein-protein interactions (PPIs) involving 34 secreted Mtb proteins. This interaction map revealed two new factors involved in Mtb pathogenesis - the secreted Mtb protein, LpqN, and its binding partner, the human ubiquitin ligase CBL. We discovered that an IpqN Mtb mutant is attenuated in macrophages, but growth is restored when $\mathrm{CBL}$ is removed. Conversely, $\mathrm{Cb}^{-/}$macrophages are resistant to viral infection, indicating that $\mathrm{CBL}$ regulates cell-intrinsic polarization between anti-bacterial and anti-viral immunity. Collectively, these findings illustrate the utility of this Mtb-human PPI map as a resource for developing a deeper understanding of the intricate interactions between $M t b$ and its host. 


\section{INTRODUCTION}

Mycobacterium tuberculosis (Mtb) infection persists as a leading cause of death worldwide, with an estimated 2 billion people chronically infected and 1-2 million deaths annually (Zumla et al., 2015). The only vaccine, the live-attenuated Bacillus Calmette-Guerin strain developed nearly 100 years ago, offers very limited protection against Mtb (Mangtani et al., 2014). In addition, current treatment is cumbersome, requiring administration of multiple, potentially toxic antibiotics over a period of months (Gillespie et al., 2014). Thus, there remains a critical need to elucidate the mechanisms by which $M t b$ disrupts the host immune response in order to both optimize vaccine strategies as well as to explore therapies that promote sterilizing host immunity as an adjunct to antibiotics.

Mtb infection begins when airborne bacilli are inhaled and phagocytosed by alveolar macrophages (Torrelles and Schlesinger, 2017). This activates pattern-recognition receptors that bind bacterial constituents, leading to expression of proinflammatory cytokines such as interleukin 1 (IL-1) and tumor necrosis factor alpha (TNF- $\alpha$ ) that are important for Mtb control (Mayer-Barber and Sher, 2015). Surprisingly, Mtb infection also activates secretion of Type-I interferons such as interferon beta (IFN- $\beta$ ) (Manzanillo et al., 2012), a hallmark cellular response to viral infection (McNab et al., 2015). IFN- $\beta$ induction has been linked to Mtb's ability to perforate the phagosome membrane through its Type VII/ESX-1 protein secretion system (Manzanillo et al., 2012; Novikov et al., 2011; Siméone et al., 2015; van der Wel et al., 2007), allowing communication between the bacterium and the host cytosol. This allows recognition of extracellular Mtb genomic DNA by cyclic GMP-AMP synthase (cGAS) (A. C. Collins et al., 2015; Wassermann et al., 2015; Watson et al., 2015; Wiens and Ernst, 2016) which activates interferon regulatory factor 3 (IRF3) to initiate IFN- $\beta$ transcription. In vivo, IFN- $\beta$ signaling counteracts anti-Mtb immunity (Manca et al., 2004; Manzanillo et al., 2012; Stanley et al., 2007), in-part by antagonizing the effects of interleukin-1 (IL-1) mediated resistance (Mayer-Barber et al., 2014). Moreover, elicitation of antiviral programs may serve to impede antibacterial 
responses of the host.

While cytokines such as IL-1, TNF- $\alpha$, and interferon gamma (IFN- $\gamma$ ) are critical for activating macrophages to control Mtb growth (Mayer-Barber and Sher, 2015), the cell intrinsic mechanisms by which macrophages restrict $M t b$ are incompletely understood (Braverman and Stanley, 2017). For example, macrophages encode an array of activities capable of eliminating bacteria, including phagocytosis and subsequent delivery to toxic lysosomes (Alonso et al., 2007; Shiloh et al., 1999). Mtb has evolved the remarkable ability to replicate within this hostile environment as it is not only intrinsically resistant to low $\mathrm{pH}$ and oxidative damage (Darwin et al., 2003; Vandal et al., 2009), but can also inhibit fusion between phagosomes and lysosomes (Armstrong and Hart, 1971; Clemens, 1996; Rohde et al., 2007). Likewise, the mechanisms by which Mtb thwarts macrophage resistance mechanisms are unclear. Many bacterial pathogens inject secreted effector proteins into host cells, often to directly interact with host proteins and disrupt host cell function (Byndloss et al., 2017). The ability of Mtb to permeabilize its phagosome via ESX-1 and communicate with the host cell cytoplasm raises the possibility that it may similarly introduce secreted effectors to target host proteins. While elegant genetic studies have implicated dozens of secreted Mtb factors in virulence (Sassetti and Rubin, 2003; Y. J. Zhang et al., 2013), whether these factors directly interact with host molecules to influence macrophage physiology is unknown.

Identifying the set of physical interactions between secreted Mtb and human proteins that occur in vivo will be crucial in understanding Mtb pathogenesis and may provide new approaches to combat infection. However, only a handful of Mtb-host protein-protein interactions (PPIs) have been characterized. For example, a previous yeast 2-hybrid screen targeting Mtb proteins identified an interaction between EsxH and the human ESCRT machinery (Mehra et al., 2013). Additional focused studies on individual Mtb factors have also identified interactions with the host proteins VPS33B and TLR2 (Bach et al., 2008; Pathak et al., 2007). However, the 
contribution of these interactions to Mtb virulence remains unclear either because of the known pleiotropic effects the bacterial factors have on the physiology of Mtb independent of the host (Siegrist et al., 2009), or because genetic disruption of the factors involved does not diminish Mtb virulence (Grundner et al., 2008).

Unbiased approaches for characterizing PPIs using mass spectrometry represent powerful ways to probe complex biological systems in an unbiased fashion (Beltrao et al., 2010; S. R. Collins et al., 2007; Krogan et al., 2006). Previously, using affinity tag purification combined with mass spectrometry (AP-MS), we have systematically identified novel, and unanticipated, host pathways required for virulence of a number of important human viruses (Shah et al., 2015), including human immunodeficiency virus (HIV) (Jäger et al., 2012), hepatitis C virus (HCV) (Ramage et al., 2015), and Kaposi's sarcoma herpes virus (KSHV) (Davis et al., 2015). Likewise, by focusing on a set of Chlamydia trachomatis virulence factors secreted into host cells, we uncovered a new pathway by which this difficult and persistent bacterial pathogen manipulates host cells (Elwell et al., 2017; Mirrashidi et al., 2015).

Here we report the results of a similar AP-MS study to identify the PPIs between Mtb secreted proteins and host factors. Using a combination of MS and bioinformatic analysis, we identified highly-specific physical interactions with host proteins and coupled this with genetics to begin to probe this PPI network. We have not only uncovered a novel secreted Mtb virulence factor essential for Mtb growth in vivo, LpqN, but have also identified the interacting host CBL ubiquitin ligase as a new restriction factor that regulates cell-intrinsic Mtb control. Surprisingly, while CBLdeficient macrophages are more permissive for Mtb growth, they are resistant to viral replication, indicating a role in modulating intrinsic control of anti-viral versus anti-bacterial responses during Mtb infection. Collectively, these findings demonstrate the value of our Mtbhuman PPI map as a resource to advance our understanding of the complex interaction between Mtb and its host. 


\section{RESULTS}

\section{Creation of an Mtb-host PPI network}

To explore the mechanisms by which Mtb disrupts host immune function, we sought to identify physical interactions between secreted Mtb factors and host proteins using a systematic proteomic approach. We reasoned that since the Mtb-containing phagosome is permeabilized (Manzanillo et al., 2012; Novikov et al., 2011; Siméone et al., 2015; van der Wel et al., 2007), any protein secreted by the bacterium would have the potential opportunity to interact with host proteins in the cell. We began by analyzing highly controlled culture filtrates of $M t b$, free of detectable cytoplasmic contamination. Using mass spectrometry (MS), we established a highconfidence set of bona-fide secreted proteins (Figure S1A; Table S1). These data were further curated with additional published MS studies of Mtb protein localization (Målen et al., 2007). These conservative criteria, requiring direct biochemical evidence of secretion beyond the bacterial cell wall, were employed to minimize inclusion of non-secreted proteins, though it precludes analysis of secreted proteins present below the level of detection by MS. These analyses identified a high-confidence set of 105 secreted $M t b$ proteins, including 69 proteins with canonical Sec-transporter N-terminal signal sequences and $21 \mathrm{ESX}$-system substrates (Table S1).

We next expressed each of these proteins in human cells as C-terminal Strep-tag fusions and identified co-purifying host proteins by MS. As expected, expression in 293T cells of the Secdependent substrates containing their natural signal sequences led to co-localization with the endoplasmic reticulum (Figure S1B). Thus, we expressed the protein predicted to correspond to the signal peptidase-processed form released by Mtb during natural infection, and determined that these factors primarily localized to the cytoplasm (Figure S1B). We initially sought to express the entire set of $M t b$ secreted proteins directly in macrophages but, consistent with reports from other groups (X. Zhang et al., 2009), found this to be an inefficient process that 
yielded insufficient protein for MS analysis (Figure S1C). To circumvent this issue, yet still allow capture of macrophage-specific factors, we developed a two-step "pull-down" strategy (Figure 1A). After initially expressing and isolating the bacterial factors from $293 \mathrm{~T}$ cells on Strept-actin resin, the immobilized proteins were then incubated in an in vitro binding reaction with lysate from differentiated human U937 macrophages before subsequent AP-MS. We found this method to be robust, with the ability to readily express and purify 99 of the 105 bacterial proteins (Figure S1D). We analyzed a subset of bacterial factors both with and without addition of U937 lysate, and found a substantial number of additional interactions specific to the macrophage lysate (Table S2).

To identify the set of interacting human proteins, we performed AP-MS in triplicate for each bacterial protein with the addition of GFP-Strep as a control (see Table S3 for raw MS data). To identify specific interactions, we utilized the MiST bioinformatic algorithm, which scores each interaction for specificity, reproducibility, and abundance using principal component analysis (Verschueren et al., 2015). Since no 'gold-standard' set of known host-Mtb protein interactions existed with which to optimize a score threshold, we used the previously-validated score threshold of 0.7 to define high-confidence interactions (Jäger et al., 2012). Using MiST, the initial interaction dataset was distilled to a high-confidence network between 34 bacterial proteins and 187 specifically-interacting human proteins (Figure 1C; Figure 3; Table S4). As a control, when the AP-MS data were randomized and then analyzed by MiST, only 3 human proteins exceeded the MiST 0.7 threshold (Figure 1B), providing further support that we defined a high quality set of PPIs. To independently validate these interactions, we defined a highpriority set of 34 host interactors with annotated immune-related functions and expressed them in 293 T cells as 3xFLAG-tagged proteins with their Strep-tagged bacterial partner. Reciprocal co-immunoprecipitation of the tagged human proteins with their putative bacterial interaction partner verified 25 of 34 (74\%) of the interactions, underscoring the reliability of AP-MS coupled with bioinformatic analysis (Figure S1E). 


\section{Features of the Mtb-Human PPI Map}

Bioinformatic analyses of the interactome revealed several striking features of the Mtb-host interaction. We found that only $34 \%$ of the Mtb proteins we targeted provided high-scoring PPIs with host proteins, which is in contrast to our previous findings with viral proteomes - HIV (89\%), HCV (100\%), and KSHV (75\%) (Davis et al., 2015; Jäger et al., 2012; Ramage et al., 2015). This suggests that, unlike viruses that are completely dependent on host functions for replication, many of the $M t b$-secreted proteins are involved in bacterial cell-autonomous processes. While we found $C$. trachomatis also had a high percentage of proteins with host interactions $(66 \%)$, this could reflect the fact that the subset of virulence factors investigated was enriched for mediating contacts with host cells (Mirrashidi et al., 2015). Second, comparison of the host-pathogen interactomes of Mtb, HIV, KSHV and C. trachomatis revealed that while there was some overlap for the host proteins bound by the different pathogens, the majority of proteins in each of the datasets were pathogen-specific. In particular, 138 of 187 host proteins in the Mtb-host interaction map are Mtb-specific (Figure 2A, Table S5). However, comparison of the functional pathways represented by these interactions revealed several commonalities between the pathogens (Figure 2B, Table S6). In particular, both Mtb and C. trachomatis datasets were enriched for proteins involved in vesicular transport, consistent with their common intracellular lifestyles. Moreover, despite some commonalities between the datasets, the interactomes reflected distinct host pathways, suggesting that these pathogens have evolved unique strategies to establish a replicative niche.

\section{Evolutionary Analysis of the Mtb-host interactome}

Our data also offer an opportunity to empirically test evolutionary hypotheses regarding Mtb and its host. There are numerous examples of host anti-viral restriction factors that are inhibited by direct binding of viral effector proteins. As a consequence, many of these restriction factors experience selective pressure to diversify rapidly (positive selection) and escape the effects of 
the viral protein - an example of the "Red Queen Hypothesis" of coevolution (Duggal and Emerman, 2012). We hypothesized that if secreted Mtb effectors physically interact with host proteins, then these host proteins might show similar evidence of positive selection. We began by using SnIPRE (Eilertson et al., 2012), which compares the relative rate of non-synonymous substitutions between human and chimpanzee orthologs and detects positive selection over a 57 million-year span, but identified no evidence of selective pressure over this timeframe (Figure $2 \mathrm{C}$, left panel). However, examination over more recent evolution within the human lineage itself (10,000-30,000-year span), revealed a significant amount of recent diversification (Figure 2C, right panel). In the human population, positively selected alleles quickly sweep to high frequency before recombination separates these variants from the surrounding genome. The integrated Haplotype Score (iHS) measures haplotype block lengths to identify variant alleles undergoing positive selection (Szpiech and Hernandez, 2014; Voight et al., 2006). In contrast to our findings over longer evolutionary timeframes, iHS detected a shift in the distribution of evolutionary rates, with increased diversification for the set of $M t b$-interacting host proteins (Figure 2C, Table S7). To control for detection bias in our MS methods, we analyzed the set of non-interacting proteins identified in our affinity purifications and found no such shift. We also analyzed the interactomes of other pathogens and found rapid diversification in HIV-interacting host proteins, but not in the interactomes of pathogens associated with lower mortality - KSHV and C. trachomatis. Thus, our findings are unlikely to be the result of a bias introduced by either MS detection or bioinformatic filtering. Rather, our data suggest that the set of $M t b$-interacting host proteins are rapidly diversifying, potentially as a result of their interactions with $M t b$ proteins.

\section{Topology of the Mtb-host interactome}

Individual proteins often associate into larger protein complexes to carry out all cellular processes (Alberts, 1998). In an effort to make the Mtb-host PPI map more interpretable, we used previously published host PPI data to arrange the identified host proteins into complexes. To this end, we used data from the CORUM and STRING databases to overlay known human 
PPIs within the Mtb interactome (Figure 3). This analysis revealed a number of host complexes targeted by Mtb proteins, including a connection between Apa and five subunits of the COP II vesicular trafficking complex as well as an association with the uncharacterized Mtb protein, Rv3722c and seven components of the CCT chaperone complex. Furthermore, we uncovered a connection between Rv1804c and Rv1075c and the STRIPAK signal transduction complex, which has been recently linked to innate immunity and autophagy targeting (Liu et al., 2016; Neisch et al., 2017). Interestingly, Rv1075c also interacts with components of the SET1 histone methyltranferase complex, COMPASS, which regulates transcriptional elongation by RNAPII (Krogan et al., 2003). This connection suggests that Mtb may regulate host transcriptional regulation by hijacking this complex using one of its secreted proteins.

Recently, our groups carried out a global analysis of changes in the host proteome with respect to post-translational modifications, including phosphorylation and ubiquitination, in the presence of Mtb infection (Table S8). Overlaying this dataset with our PPI map revealed that we uncovered 22 and 12 host proteins with altered phosphorylation and ubiquitylation, respectively (Figure 3). These data suggest that these proteins might both be regulated by the host in response to $M t b$, and targeted by $M t b$ effectors making them high-priority targets for future study.

\section{LpqN is a novel Mtb virulence factor}

To begin to identify the functionally relevant interactions between $M t b$ secreted proteins and macrophages, we used a genetic approach to disrupt bacterial and host components of the PPI map and determined the effects during infection. Initially, we selected a set of 10 bacterial factors whose host interactors had known immune-related functions or were post-translationally modified in response to Mtb infection, and we generated or obtained previously-created Mtb mutants with these genes disrupted. These mutants were evaluated for growth in primary macrophages, and four strains with impaired growth relative to wild-type Mtb were carried 
forward for further analysis (Figure 4A, data not shown). For each mutant, we created two isogenic strains carrying either a wild-type copy of the disrupted gene under the control of its native promoter on an integrated plasmid, or an empty control plasmid. Each plasmid also contained a unique DNA barcode that allowed us to determine the relative abundance of each of the eight strains within pooled infections. We infected mice with this mixture of strains via the aerosol route, recovered bacilli from lung homogenates at multiple time-points, and quantified the relative proportion of each strain by qPCR (Figure 4A). Three of the mutants competed equally with their cognate "complementation" strains, but the IpqN::Tnhimar1 mutant (IpqN mutant) was rapidly depleted relative to IpqN::Tnhimar1::plpqN (IpqN complemented).

Importantly, the IpqN mutant competed equally with the complemented strain when the pool was grown in culture (Figure S2), and the two strains grew with indistinguishable kinetics in axenic culture (Figure 4B), demonstrating that the IpqN mutant was specifically attenuated in the host.

We confirmed that the IpqN mutant was also attenuated in isolated macrophages by comparing the growth of the isogenic IpqN mutant and complemented strains. We used a bioluminescent growth assay in which the strains were transformed with the luxBCADE operon from Vibrio harveyi, and their growth in macrophages monitored by quantifying luminescence over time. As with our observations in infected mice, we found that the IpqN mutant was also significantly attenuated in primary macrophages (Figure 4C), suggesting that LpqN functions during the initial bacterial encounter with the innate immune system. Collectively, these results demonstrate that LpqN is critical for bacterial growth in both ex vivo macrophages and in mice, thus establishing LpqN as a novel Mtb virulence factor.

\section{LpqN and the ubiquitin ligase CBL interact physically and genetically.}

We hypothesized that LpqN functions to promote bacterial growth through its interaction with host proteins, either by blocking their function or by 'hijacking' them for pathogenic purposes. To test this idea, we used CRISPR/Cas9-based mutagenesis in RAW 264.7 cells to determine 
whether disruption of LpqN-interacting host factors was able to rescue the growth defect of the IpqN mutant. As noted above, our related studies using MS to examine host protein posttranslational modifications during Mtb infection demonstrated increased phosphorylation of the ubiquitin ligase $\mathrm{CBL}$, a LpqN-interacting protein, and suggested a possible role for CBL in antibacterial immunity (Figure 4D; Table S8). Indeed, while mutagenesis of several other LpqN interactors failed to rescue the IpqN mutant phenotype, we observed that disruption of $\mathrm{Cb} /$ rescued the growth of the IpqN mutant in RAW264.7 cells (Figures S3A and S3B).

We verified the physical interaction between LpqN and CBL by expressing LpqN-Strep in 293T cells. Endogenous CBL protein that copurified was detected by western blotting, verifying the interaction detected by MS (Figure 4E). Additional in vitro pull-down experiments using LpqN and $\mathrm{CBL}$ proteins produced in E. coli also revealed direct interaction between these two factors (Figure S4). We further explored the genetic interaction between IpqN and $\mathrm{Cb} /$ using primary bone marrow-derived macrophages (BMMs) deficient in CBL. To avoid the potential confounding effects of the two related CBL family ubiquitin ligases, we analyzed BMMs lacking the $C b / B$ and $C b / C$ genes, and carrying either a wild-type or floxed $C b /$ locus that could be deleted with $\sim 90 \%$ efficiency by addition of 4-hydroxy-tamoxifen to the culture media - hereafter designated $\mathrm{Cbl}^{+/+}$and $\mathrm{Cbl}^{-/}$respectively (Figure 5I)(Mukhopadhyay et al., 2016). We infected $\mathrm{Cbl}^{+/+}$and $\mathrm{Cbl}^{-/}$primary macrophages with the IpqN mutant strain and monitored bacterial growth over time. Consistent with our observations in $\mathrm{Cb}^{-1-}$ RAW264.7 cells (Figure S3B), the IpqN mutant was markedly attenuated in $\mathrm{Cb}^{I^{+/}} \mathrm{BMMs}$ (Figure 4F). Importantly, in $\mathrm{Cb}^{I_{-}} \mathrm{BMMs}$, we observed significant rescue of the IpqN mutant intracellular growth phenotype, with a 15-fold increase in bacterial growth (Figure 4F). This result was further confirmed by plating macrophage lysates and directly enumerating bacterial colony-forming units (CFU) (Figure $\mathrm{S3C})$. We also noted that both the IpqN complemented strain and the parental WT CDC1551 Mtb strain grew $\sim 2$-fold faster in $\mathrm{Cbl}^{-/-}$macrophages, indicating that $\mathrm{CBL}$ imposes some restriction on wild-type $M$. tuberculosis (Figures 4G, 4H, S3D), however, the effect of CBL 
activity was much more pronounced with the IpqN mutant (Figure 4I). Importantly, the growth of an attenuated ESX-1 mutant of $M t b$, which is unable to permeabilize its phagosome, was unaffected in $\mathrm{Cbl}^{--}$macrophages (Figure 5G), as were the growth of other intracellular bacteria including Listeria monocytogenes and Salmonella enterica serovar Typhimurium (S.

Typhimurium, Figures S3E and S3F). Thus, there is a genetic interaction between bacterial IpqN and host $\mathrm{Cbl}$, whereby growth of the IpqN mutant strain is selectively enhanced upon loss of host $C b l$, but strains expressing $\mathrm{LpqN}$ are relatively insensitive to $\mathrm{CBL}$ activity. This genetic interaction suggests that $\mathrm{LpqN}$ and $\mathrm{CBL}$ may lie in a common pathway, and collectively the combined biochemical and genetic data suggest a model whereby CBL acts as a host restriction factor, limiting Mtb growth, and LpqN acts as a virulence factor to block the normal functions of $\mathrm{CBL}$ and promote bacterial replication.

\section{CBL regulates the balance between cell-intrinsic anti-bacterial and anti-viral responses.}

CBL has been well-characterized as a ubiquitin ligase responsible for ubiquitylation and degradation of activated receptor protein tyrosine-kinases (Levkowitz et al., 1999). Since ubiquitylated proteins localize to the Mtb phagosome and are proposed to function as a targeting mechanism for autophagy-mediated host defense (Ponpuak et al., 2010; Watson et al., 2012), we initially hypothesized that CBL might directly contribute to this process. The frequency of ubiquitin colocalization with the $M t b$ phagosome after $4 \mathrm{~h}$ of infection was unaffected in $\mathrm{Cbl}^{--}$BMMs (Figure 5A). However, to more broadly evaluate the efficiency by which $\mathrm{Mtb}$ is delivered to the lysosome, we infected $\mathrm{Cbl}^{+/+}$and $\mathrm{Cbl}^{-l_{-}} \mathrm{BMMs}$ with the IpqN mutant and quantified colocalization of bacteria with the vacuolar ATPase. After $24 \mathrm{~h}$ of infection, we observed a significant decrease in colocalization with the vacuolar ATPase in $\mathrm{Cbl}^{\text {- }} \mathrm{BMM}$ (Figure $5 B$ ), consistent with the idea that $\mathrm{CBL}$ promotes innate bacterial restriction mechanisms of naïve macrophages.

Given the role of $\mathrm{CBL}$ as a regulator of signaling, we also investigated whether the ligase 
modulates the macrophage response to bacterial infection by monitoring expression of key cytokines activated in response to Mtb. Though we observed no difference in TNF- $\alpha$ mRNA levels between $\mathrm{Cbl}^{+/+}$and $\mathrm{Cbl}^{-l_{-}}$cells (Figure $5 \mathrm{C}$ ), we noted a dramatic increase in the expression of the anti-viral cytokine IFN- $\beta$ (Figure 5D). Likewise, IFIT1, another target of antiviral signaling, was also increased in $\mathrm{Cbl}^{-/-}$cells (Figure $5 \mathrm{C}$ ). Thus, the ability of $\mathrm{Mtb}$ to activate antiviral responses, likely through the exposure of bacterial DNA to the cytoplasmic sensor cGAS, is amplified in the absence of CBL, indicating that this ligase functions to negatively regulate these responses.

These data support the notion that CBL-deficient macrophages are skewed towards more robust anti-viral responses, which come at the cost of decreased anti-bacterial resistance. A prediction of this hypothesis is that $\mathrm{Cbl}^{-/-}$cells would be resistant to viral infection. To test this, we infected $\mathrm{Cbl}^{-/}$and $\mathrm{Cbl}^{+/+} \mathrm{BMMs}$ with Sendai Virus (SeV) and Herpes Simplex Virus 1 (HSV-1) and evaluated the release of viral particles $24 \mathrm{~h}$ after infection. Deletion of $\mathrm{Cb} /$ conferred resistance to both viruses, with fewer viral particles released, and a reduction in viral cytopathic effects (Figure 5E, 5F). Thus, while loss of CBL results in increased sensitivity to Mtb infection, it also creates a more restrictive environment for viral replication. This is consistent with recent findings that siRNA-mediated depletion of CBL results in stabilization of IRF3 and increased anti-viral signaling (Zhao et al., 2016), though when we evaluated whether CBL similarly regulated IRF3 during Mtb infection we detected no change in protein levels (Figure 5I). Although the exact mechanism by which $\mathrm{CBL}$ influences antibacterial/antiviral balance, the permissiveness of $\mathrm{Cbl}^{-/-}$macrophages for $\mathrm{Mtb}$ growth is unlikely to result from increased Type I interferon itself, as isolated macrophages deficient for Type I IFN production (Irf3 ${ }^{-/}$) or reception $\left(\right.$ Ifnar ${ }^{-/}$) are not altered in their ability to restrict Mtb replication (Manzanillo et al., 2012; Stanley et al., 2007). Thus, it appears that CBL may control a broader cell-intrinsic anti-bacterial program by inhibiting anti-viral responses, with one component being Type I IFN activation. 
Although the exact role of ESX-1 in Mtb virulence remains mysterious, it is generally assumed that the poor growth of ESX-1 mutants in macrophages is due primarily to their inability to perforate phagosomal membranes and access the cytosol to acquire nutrients and deliver effectors (Stanley and Cox, 2013). However, given the reciprocal effects of antiviral and antibacterial responses mediated by $\mathrm{CBL}$, it is also possible that the inability to activate the CBL-regulated antiviral program directly contributes to the attenuated phenotype of ESX-1 mutants. As discussed above, when we infected BMMs with an ESX-1 mutant, we observed no effect of $C B L$ on bacterial growth (Figure $5 G$ ). This supports the idea that $C B L$ acts by suppressing the cellular anti-viral response, and that in the absence of an anti-viral signal, CBL is no longer necessary to control the growth of ESX-1 mutants. To further investigate the idea that a CBL-regulated anti-viral program promotes Mtb growth, we tested whether ectopic activation of anti-viral responses by experimentally delivering DNA to the host cell cytosol would rescue the growth defects of ESX-1 mutant bacteria. In this way, activation of the anti-viral pathway negatively regulated by CBL would occur in the absence of phagosomal permeabilization, effectively bypassing the ESX-1 requirement. In macrophages transfected with DNA we observed a transient rescue of the ESX-1 mutant with impaired bacterial killing over the first 24h, an effect most notable in $\mathrm{Cb}^{-/}$macrophages (Figure $5 \mathrm{H}$ ). This suggests that anti-viral signaling is sufficient to blunt the anti-bacterial activity of macrophages and that CBL functions to counteract this effect. Importantly, addition of IFN- $\beta$ did not rescue growth of the ESX-1 mutant, consistent with the idea that $\mathrm{CBL}$ regulates an anti-viral program that is independent of Type I IFN.

\section{DISCUSSION}

Despite the impact of TB on mankind, surprisingly little is known about the physical interactions between Mtb proteins and its human host proteome. Our understanding of how many enteric pathogens interact with host cells via secretion of virulence factors that target host pathways has shed light on the evolution of intricate interactions that underlie the host-pathogen interface. 
Because Mtb is a remarkably successful bacterial pathogen, capable of persisting for the lifetime of the host, it seems likely that Mtb would similarly introduce secreted effectors to manipulate host pathways and forestall host immunity. However, the evidence that Mtb actually employs this strategy is scant. There are only a handful of characterized interactions between Mtb and host proteins, and it remains unclear how they impact Mtb virulence. We have addressed this question using our AP-MS pipeline to globally identify 187 high confidence, physical interactions between Mtb and host proteins. It is likely that not all of these will contribute significantly to bacterial virulence during the course of a natural Mtb infection. However, this proteomic approach, when combined with genetic analysis, represents a powerful way to identify new, biologically relevant interactions between pathogens and their host cells. Indeed, with this approach we have identified a new Mtb virulence factor, LpqN, and additionally have identified the CBL ubiquitin ligase as a protein that interacts both physically and genetically with LpqN and functions as a host restriction factor for Mtb, limiting bacterial growth in macrophages. We expect that further mining of this interaction map will reveal further interactions that underlie Mtb pathogenesis.

CBL had not been implicated in innate responses to bacteria, and the mechanisms by which it contributes to host immunity await discovery. Ubiquitin ligases play key roles in targeting of intracellular pathogens to autophagy, but our data indicates that CBL plays a regulatory role modulating cell-intrinsic responses to infection. Indeed, we noted a surprising increase in the cellular anti-viral response in the absence of $\mathrm{CBL}$, with a concomitant decrease in the efficiency of anti-bacterial processes such as phagosome-lysosome fusion. Importantly, CBL had no effect on the growth of an ESX-1 mutant strain of Mtb that does not trigger anti-viral responses. This finding that the anti-bacterial properties of $\mathrm{CBL}$ are only manifest in the setting of an anti-viral host response is consistent with the hypothesis that $\mathrm{CBL}$ is acting as a regulator of the anti-viral response to control bacterial infection. 
The specific antibacterial pathways regulated by $C B L$ in the context of Mtb infection remain unknown. IFN- $\beta$ has been suggested to impair clearance of the related pathogen Mycobacterium leprae (Teles et al., 2013), and disruption of either IRF3 or IFNAR have previously been shown to modestly impact Mtb growth in mice (Manzanillo et al., 2012; Stanley et al., 2007). However, IFN- $\beta$ signaling itself does not alter Mtb growth cell-intrinsically in isolated macrophages, as we observe with CBL. This suggests additional, uncharacterized antiviral pathways exist, and are activated by $M t b$, in addition to the known IRF3-IFN- $\beta$-IFNAR pathway. The existence of additional anti-viral pathways is further supported by our findings in a related study using proteomics to examine host protein post-translational modifications during Mtb infection (Parry et. al. in preparation). In that work, we identified the IRF7-regulated antiviral response as a potent facilitator of $M t b$ replication. The effects mediated by IRF7 and CBL share several similarities. Unlike the IRF3-IFNAR pathway, they both alter Mtb replication cellintrinsically in isolated macrophages. The effects of perturbing IRF7 and CBL are also both specific to $M t b$, as the replication of neither S. Typhimurium nor L. monocytogenes are altered. It remains to be determined whether CBL and IRF7 are actually functioning in the same pathway, or whether they modulate parallel anti-viral pathways. The finding that two different regulators of the macrophage anti-viral response both impact the ability of $M t b$ to replicate inside macrophages suggests that this is an essential element in TB pathogenesis. We postulate that Mtb might have evolved the ability to release bacterial DNA into the host in order to trigger the macrophage anti-viral response, and that this anti-viral response antagonizes antibacterial activity. Effectors such as LpqN could then potentially function to prolong or amplify such a signal and create a more permissive intracellular environment for bacterial growth.

From within its replicative niche in host macrophages, Mtb is able to undermine the host immune response and establish a chronic, often lifelong infection - but how it actually does so remains mysterious. The interaction map reported here represents a unique resource to generate testable hypotheses regarding the Mtb-host interface and to identify the host pathways 
bioRxiv preprint doi: https://doi.org/10.1101/202598; this version posted October 17,2017 . The copyright holder for this preprint (which was not certified by peer review) is the author/funder. All rights reserved. No reuse allowed without permission.

that dictate TB pathogenesis. 


\section{FIGURE LEGENDS}

Figure 1. AP-MS experimental design and analysis.

(A) Flow-chart of the AP-MS proteomics pipeline used for this study.

(B) Distribution of MiST scores for all of the interactions in the Mtb-host PPI network, as well as for a randomized control network.

(C) Number of high-confidence host interactors (MiST score $>0.7, x$-axis) for each of the 34 bacterial proteins that had at least one host interaction (y-axis). Genes encoding bacterial proteins likely essential for Mtb growth are denoted by red font (Griffin et al., 2011). See also Figure S1.

Figure 2. Secreted Mtb proteins interact with a distinct, rapidly-evolving set of human proteins.

(A) Venn diagram representing the overlap of human proteins identified in four pathogen-host PPI maps. The significance of the overlap in the PPI networks between pairwise comparisons of Mtb with HIV, C. trachomatis, and KSHV is $p=1 \times 10^{-9}, p=2.1 \times 10^{-5}$ and $p=0.008$ respectively, using Fisher's exact test. This data is also presented in Table S7.

(B) Host biological processes enriched in each PPI map for Mtb, C. trachomatis, HIV, and KSHV. Processes enriched $>2$-fold with $p$-value $<0.05$ after Benjamini-Hochberg multiple comparisons correction, are displayed.

(C) Evolutionary rates of Mtb-interacting proteins within the chimpanzee lineage using SniPRE (left) and within the human lineage using iHS analysis (right). The distributions of evolutionary scores across host proteins partitioned into two groups: Mtb-interacting proteins shown in blue and non-interacting proteins shown in white, with higher values indicating diversifying selection. Analysis of HIV, Chlamydia and KSHV interactomes are shown for comparison. Solid line denotes median, dashed lines denote upper/lower quartile. ${ }^{*} p=0.02,{ }^{* *} p<0.01$.

\section{Figure 3. Mtb-host protein-protein interaction network}

A network representation of the $34 \mathrm{Mtb}$ proteins (yellow circles) and 187 human proteins (light blue circles), with blue edges representing the interactions identified in this study. Humanhuman interactions (thin grey lines) were defined by the CORUM and STRING databases, with known protein complexes highlighted in green. Proteins differentially phosphorylated and/or ubiquitylated upon Mtb infection are indicated by concentric circles.

Figure 4. The LpqN-Interacting protein CBL is a host restriction factor for Mtb.

(A) In vivo competition assay. Bacterial mutant strains (LpqN/Rv0583c, Rv2469c, Rv1804c, Rv0999) and cognate complemented strains were pooled and used to infect mice via the respiratory route. At the indicated times, bacteria were recovered from lung homogenates and the relative proportion of each strain was quantified by qPCR using unique sequence tags present in each strain.

(B) Growth curve of the indicated strains in standard $7 \mathrm{H} 9$ mycobacterial media. Representative data from two independent experiments is shown.

(C) Luminescent bacterial growth assay. BMMs were infected with the indicated strains carrying the $L U x B C A D E$ reporter operon at an effective $M O I=1$. Relative luminescent units (RLU) were quantified at the indicated times and mean $R L U$ relative to $t=0$ is plotted. Mean $\pm S E M$ are displayed from four replicate samples. Representative data of three independent experiments are shown. * $p<0.05$ by t-test.

(D) Phosphoproteomic analysis. RAW264.7 cells were isotopically labeled and infected with the indicated bacteria. Lysates were analyzed by quantitative LC-MS-MS and the fold-increase for the CBL S450 phosphosite is shown. Mean \pm SEM are displayed for two biological replicates, each with two technical replicates.

(E) LpqN-Strep or GFP-Strep was expressed in 293T cells and purified with Strep-tactin resin under native conditions, followed by SDS-PAGE and western blotting using antibodies that recognize $\mathrm{CBL}$.

(F-H) Luminescent bacterial growth assay. $\mathrm{CbI}^{{ }^{--}}$and $\mathrm{Cbl}^{\mathrm{I}^{++}} \mathrm{BMMs}$ were infected with the IpqN 
mutant (F), IpqN complemented (G), and WT Mtb CDC1551 strain (H). Mean \pm SEM of four replicate samples is displayed. Representative data from two independent experiments is shown.

(I) Restriction by CBL was derived by determining the ratio of bacterial growth in $\mathrm{Cbr}^{/-}$and $\mathrm{Cbl}^{+/+}$ BMMs at the final timepoint using data from $(\mathrm{F}-\mathrm{H})$. Error bars denote SEM.

See also Figures S2, S3 and S4.

Figure 5. CBL represses anti-viral responses during $M$ tb infection.

(A) $\mathrm{Cbl}^{-/-}$and $\mathrm{Cbl}^{+/+} \mathrm{BMMs}$ infected with $\mathrm{lpqN}$ mutant for $4 \mathrm{~h}$ and immunostained for polyubiquitin. Percent colocaliztion of $>500$ phagosomes counted per condition, scored by a microscopist blinded to sample identity. Mean \pm SEM displayed.

(B) BMMs infected with IpqN mutant $M t b$ for $24 \mathrm{~h}$ and immunostained for vacuolar ATPase. (C-D) BMMs infected with IpqN mutant Mtb for 6 hours and analyzed by RT-qPCR for the proinflammatory cytokine TNF- $\alpha(C)$, or the anti-viral response genes IFN- $\beta$ and IFIT1 (D). (E) BMMs infected with Sendai virus $(\mathrm{SeV})$ for $24 \mathrm{~h}$, and the relative number of virions in the supernatant were quantified by RT-qPCR; Mean \pm SEM displayed. Phase-contrast image (10x) taken at $24 \mathrm{~h}$ post-infection.

(F) Infection with HSV-1 for $24 \mathrm{~h}$, analyzed as in (E).

(G) Luminescent growth assay of ESX-1-deficient strain ( $\triangle E c c C-L u x B C A D E)$ in BMMs.

$(H)$ Luminescent growth assay. BMMs were treated with DNA (Lipo+DNA), transfection reagent alone (Lipo), or IFN- $\beta$ (250U) as indicated. Mean \pm SEM of four replicate samples are shown.

IFN- $\beta$ was added $4 \mathrm{~h}$ pre-infection and DNA was delivered $1 \mathrm{~h}$ post-infection. ${ }^{*} p=0.003$ by t-test

(I) BMMs were infected with IpqN mutant Mtb for $6 \mathrm{~h}$ and analyzed by Western blot.

\section{Figure 6. Model of balance between anti-viral and anti-bacterial cell-intrinsic immune response pathways.}

Host macrophages tailor responses to distinct kinds of pathogens at the earliest stages of infection by activating cell-intrinsic immune pathways tailored to the threat, e.g. virus or bacterium. These programs appear to be mutually antagonistic, as activation of antiviral pathways comes at the cost of antibacterial immunity during Mtb infection. Our work indicates that CBL functions to influence this balance by inhibiting viral responses and promoting antibacterial immunity. Macrophage plasticity can be manipulated by pathogens (dashed arrows) to tip the balance to promote inappropriate responses to favor replication.

\section{AUTHOR CONTRIBUTIONS}

B.H.P., N.J.K. and J.S.C. conceived the project; B.H.P., Z.N, J.R.J., T.P, D.A.P., R.H., L.C., N.J.K. and J.S.C. designed experiments; B.H.P., Z.N, J.R.J., J.V.D., G.M.J., T.J., Y.M.O., C.M., S.L.B., K.G., X.D., A.C., T.P., C.C., and S.J. performed the experimental work; B.H.P., Z.N, J.R.J., J.V.D., M.S., D.A.P., R.H., L.C., N.J.K. and J.S.C. analyzed the results; B.H.P., Z.N., C.M., N.J.K. and J.S.C. wrote the manuscript; M.N., D.A.P., R.H. and L.C. contributed reagents and technical expertise.

\section{CONFLICT OF INTEREST}

Daniel A. Portnoy has a financial interest in Aduro Biotech, and both he and the company stand to benefit from commercialization of this research.

\section{ACKNOWLEDGMENTS}

We thank members of the Cox, Krogan and Stanley (UCB) labs for comments on the manuscript and for invaluable discussions. This work was supported by NIH grants P01 Al063302 (N.J.K., J.S.C., D.A.P.), P50 GM082250 (N.J.K.), U19 Al106754 (N.J.K.), DP1 Al124619 (J.S.C.), and R01 Al120694 (N.J.K. and J.S.C.). B.P.H. was supported by an A.P. Giannini award and NIH K08 (K08Al104766).

\section{REFERENCES}


Alberts, B., 1998. The cell as a collection of protein machines: Preparing the next generation of molecular biologists. Cell 92, 291-294. doi:10.1016/S0092-8674(00)80922-8

Alonso, S., Pethe, K., Russell, D.G., Purdy, G.E., 2007. Lysosomal killing of Mycobacterium mediated by ubiquitin-derived peptides is enhanced by autophagy. Proc Natl Acad Sci USA 104, 6031-6036. doi:10.1073/pnas.0700036104

Armstrong, J.A., Hart, P.D., 1971. Response of cultured macrophages to mycobacterium tuberculosis, with observations on fusion of lysosomes with phagosomes. J Exp Med 134, 713740. doi:10.1084/jem.134.3.713

Bach, H., Papavinasasundaram, K.G., Wong, D., Hmama, Z., Av-Gay, Y., 2008. Mycobacterium tuberculosis virulence is mediated by PtpA dephosphorylation of human vacuolar protein sorting 33B. Cell Host \& Microbe 3, 316-322. doi:10.1016/j.chom.2008.03.008

Beltrao, P., Cagney, G., Krogan, N.J., 2010. Quantitative genetic interactions reveal biological modularity. Cell 141, 739-745. doi:10.1016/j.cell.2010.05.019

Braverman, J., Stanley, S.A., 2017. Nitric Oxide Modulates Macrophage Responses to Mycobacterium tuberculosis Infection through Activation of HIF-1 1 and Repression of NF-kB. J Immunol ji1700515. doi:10.4049/jimmunol.1700515

Byndloss, M.X., Rivera-Chávez, F., Tsolis, R.M., Bäumler, A.J., 2017. How bacterial pathogens use type III and type IV secretion systems to facilitate their transmission. Curr Opin Microbiol 35, 1-7. doi:10.1016/j.mib.2016.08.007

Clemens, D.L., 1996. Characterization of the Mycobacterium tuberculosis phagosome. Trends Microbiol 4, 113-118. doi:10.1016/0966-842x(96)81528-9

Collins, A.C., Cai, H., Li, T., Franco, L.H., Li, X.-D., Nair, V.R., Scharn, C.R., Stamm, C.E., Levine, B., Chen, Z.J., Shiloh, M.U., 2015. Cyclic GMP-AMP Synthase Is an Innate Immune DNA Sensor for Mycobacterium tuberculosis. Cell host \& microbe 17, 820-828. doi:10.1016/j.chom.2015.05.005

Collins, S.R., Miller, K.M., Maas, N.L., Roguev, A., Fillingham, J., Chu, C.S., Schuldiner, M., Gebbia, M., Recht, J., Shales, M., Ding, H., Xu, H., Han, J., Ingvarsdottir, K., Cheng, B., Andrews, B., Boone, C., Berger, S.L., Hieter, P., Zhang, Z., Brown, G.W., Ingles, C.J., Emili, A., Allis, C.D., Toczyski, D.P., Weissman, J.S., Greenblatt, J.F., Krogan, N.J., 2007. Functional dissection of protein complexes involved in yeast chromosome biology using a genetic interaction map. Nature 446, 806-810. doi:10.1038/nature05649

Darwin, K.H., Ehrt, S., Gutierrez-Ramos, J.-C., Weich, N., Nathan, C.F., 2003. The Proteasome of Mycobacterium tuberculosis Is Required for Resistance to Nitric Oxide. Science 302, 19631966. doi:10.1126/science. 1091176

Davis, Z.H., Verschueren, E., Jang, G.M., Kleffman, K., Johnson, J.R., Park, J., VonDollen, J., Maher, M.C., Johnson, T., Newton, W., Jäger, S., Shales, M., Horner, J., Hernandez, R.D., Krogan, N.J., Glaunsinger, B.A., 2015. Global mapping of herpesvirus-host protein complexes reveals a transcription strategy for late genes. Mol Cell 57, 349-360.

doi:10.1016/j.molcel.2014.11.026

Duggal, N.K., Emerman, M., 2012. Evolutionary conflicts between viruses and restriction factors shape immunity. Nat Rev Immunol 12, 687-695. doi:10.1038/nri3295

Eilertson, K.E., Booth, J.G., Bustamante, C.D., 2012. SnIPRE: selection inference using a Poisson random effects model. PLoS Comput Biol 8, e1002806.

doi:10.1371/journal.pcbi.1002806

Elwell, C.A., Czudnochowski, N., Dollen, Von, J., Johnson, J.R., Nakagawa, R., Mirrashidi, K., Krogan, N.J., Engel, J.N., Rosenberg, O.S., 2017. Chlamydia interfere with an interaction between the mannose-6-phosphate receptor and sorting nexins to counteract host restriction. 
Elife 6, 213. doi:10.7554/eLife.22709

Gillespie, S.H., Crook, A.M., McHugh, T.D., Mendel, C.M., Meredith, S.K., Murray, S.R., Pappas, F., Phillips, P.P.J., Nunn, A.J., REMoxTB Consortium, 2014. Four-month moxifloxacinbased regimens for drug-sensitive tuberculosis. N Engl J Med 371, 1577-1587. doi:10.1056/NEJMoa1407426

Griffin, J.E., Gawronski, J.D., Dejesus, M.A., loerger, T.R., Akerley, B.J., Sassetti, C.M., 2011. High-resolution phenotypic profiling defines genes essential for mycobacterial growth and cholesterol catabolism. PLoS Pathog 7, e1002251. doi:10.1371/journal.ppat.1002251

Grundner, C., Cox, J.S., Alber, T., 2008. Protein tyrosine phosphatase PtpA is not required for Mycobacterium tuberculosis growth in mice. FEMS Microbiol Lett 287, 181-184.

doi:10.1111/j.1574-6968.2008.01309.x

Jäger, S., Cimermancic, P., Gulbahce, N., Johnson, J.R., McGovern, K.E., Clarke, S.C., Shales, M., Mercenne, G., Pache, L., Li, K., Hernandez, H., Jang, G.M., Roth, S.L., Akiva, E., Marlett, J., Stephens, M., D'Orso, I., Fernandes, J., Fahey, M., Mahon, C., O'Donoghue, A.J., Todorovic, A., Morris, J.H., Maltby, D.A., Alber, T., Cagney, G., Bushman, F.D., Young, J.A., Chanda, S.K., Sundquist, W.I., Kortemme, T., Hernandez, R.D., Craik, C.S., Burlingame, A., Sali, A., Frankel, A.D., Krogan, N.J., 2012. Global landscape of HIV-human protein complexes. Nature 481, 365370. doi:10.1038/nature 10719

Krogan, N.J., Cagney, G., Yu, H., Zhong, G., Guo, X., Ignatchenko, A., Li, J., Pu, S., Datta, N., Tikuisis, A.P., Punna, T., Peregrín-Alvarez, J.M., Shales, M., Zhang, X., Davey, M., Robinson, M.D., Paccanaro, A., Bray, J.E., Sheung, A., Beattie, B., Richards, D.P., Canadien, V., Lalev, A., Mena, F., Wong, P., Starostine, A., Canete, M.M., Vlasblom, J., Wu, S., Orsi, C., Collins, S.R., Chandran, S., Haw, R., Rilstone, J.J., Gandi, K., Thompson, N.J., Musso, G., St Onge, P., Ghanny, S., Lam, M.H.Y., Butland, G., Altaf-UI, A.M., Kanaya, S., Shilatifard, A., O'Shea, E., Weissman, J.S., Ingles, C.J., Hughes, T.R., Parkinson, J., Gerstein, M., Wodak, S.J., Emili, A., Greenblatt, J.F., 2006. Global landscape of protein complexes in the yeast Saccharomyces cerevisiae. Nature 440, 637-643. doi:10.1038/nature04670

Krogan, N.J., Dover, J., Wood, A., Schneider, J., Heidt, J., Boateng, M.A., Dean, K., Ryan, O.W., Golshani, A., Johnston, M., Greenblatt, J.F., Shilatifard, A., 2003. The Paf1 complex is required for histone $\mathrm{H} 3$ methylation by COMPASS and Dot1p: linking transcriptional elongation to histone methylation. Mol Cell 11, 721-729.

Levkowitz, G., Waterman, H., Ettenberg, S.A., Katz, M., Tsygankov, A.Y., Alroy, I., Lavi, S., Iwai, K., Reiss, Y., Ciechanover, A., Lipkowitz, S., Yarden, Y., 1999. Ubiquitin ligase activity and tyrosine phosphorylation underlie suppression of growth factor signaling by c-Cbl/Sli-1. Mol Cell 4, 1029-1040.

Liu, B., Zheng, Y., Yin, F., Yu, J., Silverman, N., Pan, D., 2016. Toll Receptor-Mediated Hippo Signaling Controls Innate Immunity in Drosophila. Cell 164, 406-419. doi:10.1016/j.cell.2015.12.029

Manca, C., Reed, M., Sherry Freeman, Mathema, B., Kreiswirth, B., Barry, C., Kaplan, G., 2004. Differential Monocyte Activation Underlies Strain-Specific Mycobacterium tuberculosis Pathogenesis. Infect Immun 72, 5511-5514. doi:10.1128/IAI.72.9.5511-5514.2004

Mangtani, P., Abubakar, I., Ariti, C., Beynon, R., Pimpin, L., Fine, P.E.M., Rodrigues, L.C., Smith, P.G., Lipman, M., Whiting, P.F., Sterne, J.A., 2014. Protection by BCG vaccine against tuberculosis: a systematic review of randomized controlled trials. Clinical infectious diseases: an official publication of the Infectious Diseases Society of America 58, 470-480. doi:10.1093/cid/cit790

Manzanillo, P.S., Shiloh, M.U., Portnoy, D.A., Cox, J.S., 2012. Mycobacterium tuberculosis activates the DNA-dependent cytosolic surveillance pathway within macrophages. Cell host \& 
microbe 11, 469-480. doi:10.1016/j.chom.2012.03.007

Mayer-Barber, K.D., Andrade, B.B., Oland, S.D., Amaral, E.P., Barber, D.L., Gonzales, J., Derrick, S.C., Shi, R., Kumar, N.P., Wei, W., Yuan, X., Zhang, G., Cai, Y., Babu, S., Catalfamo, M., Salazar, A.M., Via, L.E., Barry, C.E., Sher, A., 2014. Host-directed therapy of tuberculosis based on interleukin-1 and type I interferon crosstalk. Nature 511, 99-103.

doi:10.1038/nature13489

Mayer-Barber, K.D., Sher, A., 2015. Cytokine and lipid mediator networks in tuberculosis. Immunol Rev 264, 264-275. doi:10.1111/imr.12249

Målen, H., Berven, F.S., Fladmark, K.E., Wiker, H.G., 2007. Comprehensive analysis of exported proteins from Mycobacterium tuberculosis H37Rv. Proteomics 7, 1702-1718. doi:10.1002/pmic.200600853

McNab, F., Mayer-Barber, K., Sher, A., Wack, A., O'garra, A., 2015. Type I interferons in infectious disease. Nat Rev Immunol 15, 87-103. doi:10.1038/nri3787

Mehra, A., Zahra, A., Thompson, V., Sirisaengtaksin, N., Wells, A., Porto, M., Koster, S., Penberthy, K., Kubota, Y., Dricot, A., Rogan, D., Vidal, M., Hill, D.E., Bean, A.J., Philips, J.A., 2013. Mycobacterium tuberculosis type VII secreted effector EsxH targets host ESCRT to impair trafficking. PLoS Pathog 9, e1003734. doi:10.1371/journal.ppat.1003734

Mirrashidi, K.M., Elwell, C.A., Verschueren, E., Johnson, J.R., Frando, A., Dollen, Von, J., Rosenberg, O., Gulbahce, N., Jang, G., Johnson, T., Jäger, S., Gopalakrishnan, A.M., Sherry, J., Dunn, J.D., Olive, A., Penn, B., Shales, M., Cox, J.S., Starnbach, M.N., Derre, I., Valdivia, R., Krogan, N.J., Engel, J., 2015. Global Mapping of the Inc-Human Interactome Reveals that Retromer Restricts Chlamydia Infection. Cell host \& microbe 18, 109-121. doi:10.1016/j.chom.2015.06.004

Mukhopadhyay, C., Triplett, A., Bargar, T., Heckman, C., Wagner, K.-U., Naramura, M., 2016. Casitas B-cell lymphoma ( $\mathrm{Cbl}$ ) proteins protect mammary epithelial cells from proteotoxicity of active c-Src accumulation. Proc Natl Acad Sci USA 113, E8228-E8237. doi:10.1073/pnas.1615677113

Neisch, A.L., Neufeld, T.P., Hays, T.S., 2017. A STRIPAK complex mediates axonal transport of autophagosomes and dense core vesicles through PP2A regulation. J Cell Biol 216, 441-461. doi:10.1083/jcb.201606082

Novikov, A., Cardone, M., Thompson, R., Shenderov, K., Kirschman, K.D., Mayer-Barber, K.D., Myers, T.G., Rabin, R.L., Trinchieri, G., Sher, A., Feng, C.G., 2011. Mycobacterium tuberculosis triggers host type I IFN signaling to regulate IL-1 $\beta$ production in human macrophages. J Immunol 187, 2540-2547. doi:10.4049/jimmunol.1100926

Pathak, S.K., Basu, S., Basu, K.K., Banerjee, A., Pathak, S., Bhattacharyya, A., Kaisho, T., Kundu, M., Basu, J., 2007. Direct extracellular interaction between the early secreted antigen ESAT-6 of Mycobacterium tuberculosis and TLR2 inhibits TLR signaling in macrophages. Nat Immunol 8, 610-618. doi:10.1038/ni1468

Ponpuak, M., Davis, A.S., Roberts, E.A., Delgado, M.A., Dinkins, C., Zhao, Z., Virgin, H.W., Kyei, G.B., Johansen, T., Vergne, I., Deretic, V., 2010. Delivery of cytosolic components by autophagic adaptor protein p62 endows autophagosomes with unique antimicrobial properties. Immunity 32, 329-341. doi:10.1016/j.immuni.2010.02.009

Ramage, H.R., Kumar, G.R., Verschueren, E., Johnson, J.R., Dollen, Von, J., Johnson, T., Newton, B., Shah, P., Horner, J., Krogan, N.J., Ott, M., 2015. A combined proteomics/genomics approach links hepatitis $C$ virus infection with nonsense-mediated mRNA decay. Mol Cell 57, 329-340. doi:10.1016/j.molcel.2014.12.028

Rohde, K., Yates, R.M., Purdy, G.E., Russell, D.G., 2007. Mycobacterium tuberculosis and the 
environment within the phagosome. Immunol Rev 219, 37-54. doi:10.1111/j.1600065X.2007.00547.x

Sassetti, C.M., Rubin, E.J., 2003. Genetic requirements for mycobacterial survival during infection. Proc Natl Acad Sci USA 100, 12989-12994. doi:10.1073/pnas.2134250100

Shah, P.S., Wojcechowskyj, J.A., Eckhardt, M., Krogan, N.J., 2015. Comparative mapping of host-pathogen protein-protein interactions. Curr Opin Microbiol 27, 62-68.

doi:10.1016/j.mib.2015.07.008

Shiloh, M.U., Macmicking, J.D., Nicholson, S., Brause, J.E., Potter, S., Marino, M., Fang, F., Dinauer, M., Nathan, C., 1999. Phenotype of mice and macrophages deficient in both phagocyte oxidase and inducible nitric oxide synthase. Immunity 10, 29-38.

Siegrist, M.S., Unnikrishnan, M., McConnell, M.J., Borowsky, M., Cheng, T.-Y., Siddiqi, N., Fortune, S.M., Moody, D.B., Rubin, E.J., 2009. Mycobacterial Esx-3 is required for mycobactinmediated iron acquisition. Proc Natl Acad Sci USA 106, 18792-18797.

doi:10.1073/pnas.0900589106

Siméone, R., Sayes, F., Song, O., Gröschel, M.I., Brodin, P., Brosch, R., Majlessi, L., 2015. Cytosolic access of Mycobacterium tuberculosis: critical impact of phagosomal acidification control and demonstration of occurrence in vivo. PLoS Pathog 11, e1004650. doi:10.1371/journal.ppat.1004650

Stanley, S.A., Cox, J.S., 2013. Host-pathogen interactions during Mycobacterium tuberculosis infections. Curr Top Microbiol Immunol 374, 211-241. doi:10.1007/82_2013_332

Stanley, S.A., Johndrow, J.E., Manzanillo, P., Cox, J.S., 2007. The Type I IFN response to infection with Mycobacterium tuberculosis requires ESX-1-mediated secretion and contributes to pathogenesis. J Immunol 178, 3143-3152. doi:10.4049/jimmunol.178.5.3143

Szpiech, Z.A., Hernandez, R.D., 2014. selscan: an efficient multithreaded program to perform EHH-based scans for positive selection. 31, 2824-2827. doi:10.1093/molbev/msu211

Teles, R.M.B., Graeber, T.G., Krutzik, S.R., Montoya, D., Schenk, M., Lee, D.J., Komisopoulou, E., Kelly-Scumpia, K., Chun, R., lyer, S.S., Sarno, E.N., Rea, T.H., Hewison, M., Adams, J.S., Popper, S.J., Relman, D.A., Stenger, S., Bloom, B.R., Cheng, G., Modlin, R.L., 2013. Type I interferon suppresses type II interferon-triggered human anti-mycobacterial responses. Science 339, 1448-1453. doi:10.1126/science.1233665

Torrelles, J.B., Schlesinger, L.S., 2017. Integrating Lung Physiology, Immunology, and Tuberculosis. Trends Microbiol 25, 688-697. doi:10.1016/j.tim.2017.03.007

van der Wel, N., Hava, D., Houben, D., Fluitsma, D., van Zon, M., Pierson, J., Brenner, M., Peters, P.J., 2007. M. tuberculosis and M. leprae translocate from the phagolysosome to the cytosol in myeloid cells. Cell 129, 1287-1298. doi:10.1016/j.cell.2007.05.059

Vandal, O.H., Nathan, C.F., Ehrt, S., 2009. Acid resistance in Mycobacterium tuberculosis. J Bacteriol 191, 4714-4721. doi:10.1128/JB.00305-09

Verschueren, E., Dollen, Von, J., Cimermancic, P., Gulbahce, N., Sali, A., Krogan, N.J., 2015. Scoring Large-Scale affinity purification mass spectrometry datasets with MiST. Curr Protoc Bioinformatics 2015, 8.19.1-8.19.16. doi:10.1002/0471250953.bi0819s49

Voight, B.F., Kudaravalli, S., Wen, X., Pritchard, J.K., 2006. A map of recent positive selection in the human genome. PLoS Biol 4, e72. doi:10.1371/journal.pbio.0040072

Wassermann, R., Gulen, M.F., Sala, C., Perin, S.G., Lou, Y., Rybniker, J., Schmid-Burgk, J.L., Schmidt, T., Hornung, V., Cole, S.T., Ablasser, A., 2015. Mycobacterium tuberculosis Differentially Activates cGAS- and Inflammasome-Dependent Intracellular Immune Responses through ESX-1. Cell Host \& Microbe 17, 799-810. doi:10.1016/j.chom.2015.05.003 
Watson, R.O., Bell, S.L., MacDuff, D.A., Kimmey, J.M., Diner, E.J., Olivas, J., Vance, R.E., Stallings, C.L., Virgin, H.W., Cox, J.S., 2015. The Cytosolic Sensor cGAS Detects Mycobacterium tuberculosis DNA to Induce Type I Interferons and Activate Autophagy. Cell host \& microbe 17, 811-819. doi:10.1016/j.chom.2015.05.004

Watson, R.O., Manzanillo, P.S., Cox, J.S., 2012. Extracellular M. tuberculosis DNA targets bacteria for autophagy by activating the host DNA-sensing pathway. Cell 150, 803-815. doi:10.1016/j.cell.2012.06.040

Wiens, K.E., Ernst, J.D., 2016. The Mechanism for Type I Interferon Induction by Mycobacterium tuberculosis is Bacterial Strain-Dependent. PLoS Pathog 12, e1005809. doi:10.1371/journal.ppat.1005809

Zhang, X., Edwards, J.P., Mosser, D.M., 2009. The expression of exogenous genes in macrophages: obstacles and opportunities. Methods Mol. Biol. 531, 123-143. doi:10.1007/9781-59745-396-7_9

Zhang, Y.J., Reddy, M.C., loerger, T.R., Rothchild, A.C., Dartois, V., Schuster, B.M., Trauner, A., Wallis, D., Galaviz, S., Huttenhower, C., Sacchettini, J.C., Behar, S.M., Rubin, E.J., 2013. Tryptophan biosynthesis protects mycobacteria from CD4 T-cell-mediated killing. Cell 155, 1296-1308. doi:10.1016/j.cell.2013.10.045

Zhao, X., Zhu, H., Yu, J., Li, H., Ge, J., Chen, W., 2016. c-Cbl-mediated ubiquitination of IRF3 negatively regulates IFN- $\beta$ production and cellular antiviral response. 28, 1683-1693. doi:10.1016/j.cellsig.2016.08.002

Zumla, A., George, A., Sharma, V., Herbert, R.H.N., Baroness Masham of Ilton, Oxley, A., Oliver, M., 2015. The WHO 2014 global tuberculosis report--further to go. Lancet Glob Health 3, e10-2. doi:10.1016/S2214-109X(14)70361-4 
Figure 1. AP-MS experimental design and analysis.

(A) Flow-chart of the AP-MS proteomics pipeline used for this study.

(B) Distribution of MiST scores for all of the interactions in the Mtb-host PPI network, as well as for a randomized control network.

(C) Number of high-confidence host interactors (MiST score $>0.7, x$-axis) for each of the 34 bacterial proteins that had at least one host interaction (y-axis). Genes encoding bacterial proteins likely essential for Mtb growth are denoted by red font (Griffin et al., 2011). See also Figure S1. 
Figure 2. Secreted Mtb proteins interact with a distinct, rapidly-evolving set of human proteins.

(A) Venn diagram representing the overlap of human proteins identified in four pathogen-host PPI maps. The significance of the overlap in the PPI networks between pairwise comparisons of Mtb with HIV, C. trachomatis, and KSHV is $p=1 \times 10^{-9}, p=2.1 \times 10^{-5}$ and $p=0.008$ respectively, using Fisher's exact test. This data is also presented in Table S7.

(B) Host biological processes enriched in each PPI map for Mtb, C. trachomatis, HIV, and KSHV. Processes enriched $>2$-fold with $p$-value $<0.05$ after Benjamini-Hochberg multiple comparisons correction, are displayed.

(C) Evolutionary rates of Mtb-interacting proteins within the chimpanzee lineage using SniPRE (left) and within the human lineage using iHS analysis (right). The distributions of evolutionary scores across host proteins partitioned into two groups: Mtb-interacting proteins shown in blue and non-interacting proteins shown in white, with higher values indicating diversifying selection. Analysis of HIV, Chlamydia and KSHV interactomes are shown for comparison. Solid line denotes median, dashed lines denote upper/lower quartile. ${ }^{*} p=0.02,{ }^{* *} p<0.01$. 


\section{A}

M. tuberculosis

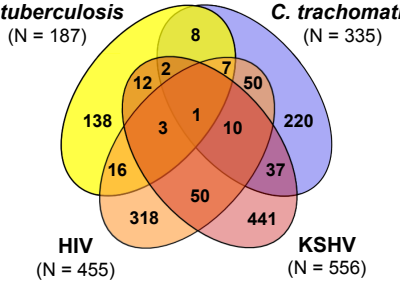

C

M. tuberculosis

C. trachomatis

KSHV

HIV

Timescale $=100 \mathrm{~K}-5 \mathrm{MYA}$

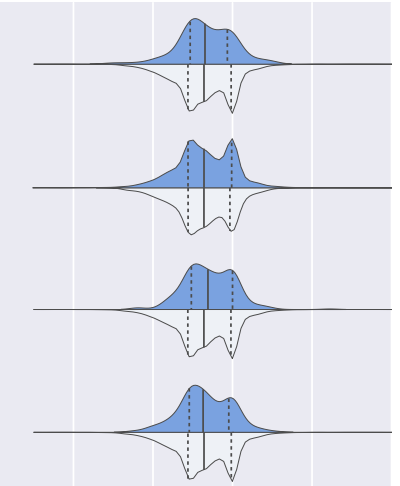

$\begin{array}{llllll}-0.6 & -0.4 & -0.2 & 0 & 0.2 & 0.4\end{array}$

SniPRE Score
B M. tuberculosis

C. trachomatis

HIV

KSHV

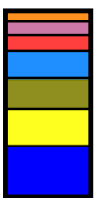

Total $=94$
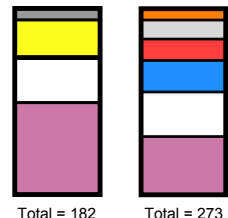

Total $=273$

Cytoskeletal regulators

Cell cycle

Endosomal/cyto vesicle transport

mRNA processing

Translation

DNA repair/recombination

ER/Golgi function Mitochondrial function Chaperone

Hydrogen ion transport

DNA damage

Transcription
Total $=260$

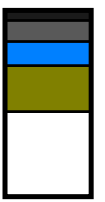


Figure 3. Mtb-host protein-protein interaction network

A network representation of the $34 \mathrm{Mtb}$ proteins (yellow circles) and 187 human proteins (light blue circles), with blue edges representing the interactions identified in this study. Humanhuman interactions (thin grey lines) were defined by the CORUM and STRING databases, with known protein complexes highlighted in green. Proteins differentially phosphorylated and/or ubiquitylated upon Mtb infection are indicated by concentric circles. 
Figure 4. The LpqN-Interacting protein CBL is a host restriction factor for Mtb. (A) In vivo competition assay. Bacterial mutant strains (LpqN/Rv0583c, Rv2469c, Rv1804c, Rv0999) and cognate complemented strains were pooled and used to infect mice via the respiratory route. At the indicated times, bacteria were recovered from lung homogenates and the relative proportion of each strain was quantified by qPCR using unique sequence tags present in each strain.

(B) Growth curve of the indicated strains in standard 7H9 mycobacterial media. Representative data from two independent experiments is shown.

(C) Luminescent bacterial growth assay. BMMs were infected with the indicated strains carrying the $L U x B C A D E$ reporter operon at an effective $M O I=1$. Relative luminescent units (RLU) were quantified at the indicated times and mean RLU relative to $t=0$ is plotted. Mean \pm SEM are displayed from four replicate samples. Representative data of three independent experiments are shown. * $p<0.05$ by t-test.

(D) Phosphoproteomic analysis. RAW264.7 cells were isotopically labeled and infected with the indicated bacteria. Lysates were analyzed by quantitative LC-MS-MS and the fold-increase for the CBL S450 phosphosite is shown. Mean \pm SEM are displayed for two biological replicates, each with two technical replicates.

(E) LpqN-Strep or GFP-Strep was expressed in 293T cells and purified with Strep-tactin resin under native conditions, followed by SDS-PAGE and western blotting using antibodies that recognize CBL.

$(\mathrm{F}-\mathrm{H})$ Luminescent bacterial growth assay. $\mathrm{Cbl}^{-\gamma^{-}}$and $\mathrm{Cbl}^{+/+} \mathrm{BMMs}$ were infected with the IpqN mutant (F), IpqN complemented (G), and WT Mtb CDC1551 strain (H). Mean \pm SEM of four replicate samples is displayed. Representative data from two independent experiments is shown.

(I) Restriction by CBL was derived by determining the ratio of bacterial growth in $\mathrm{Cbr}^{/-}$and $\mathrm{CbI}^{+/+}$ BMMs at the final timepoint using data from $(\mathrm{F}-\mathrm{H})$. Error bars denote SEM.

See also Figures S2, S3 and S4. 
Figure 5. CBL represses anti-viral responses during Mtb infection.

(A) $\mathrm{Cbl}^{-l_{-}}$and $\mathrm{Cbl}^{+/+}$BMMs infected with IpqN mutant for $4 \mathrm{~h}$ and immunostained for polyubiquitin. Percent colocaliztion of $>500$ phagosomes counted per condition, scored by a microscopist blinded to sample identity. Mean \pm SEM displayed.

(B) BMMs infected with IpqN mutant Mtb for $24 \mathrm{~h}$ and immunostained for vacuolar ATPase. (C-D) BMMs infected with IpqN mutant Mtb for 6 hours and analyzed by RT-qPCR for the proinflammatory cytokine TNF- $\alpha(C)$, or the anti-viral response genes IFN- $\beta$ and IFIT1 (D). (E) BMMs infected with Sendai virus $(\mathrm{SeV})$ for $24 \mathrm{~h}$, and the relative number of virions in the supernatant were quantified by RT-qPCR; Mean \pm SEM displayed. Phase-contrast image (10x) taken at $24 \mathrm{~h}$ post-infection.

(F) Infection with HSV-1 for $24 \mathrm{~h}$, analyzed as in (E).

(G) Luminescent growth assay of ESX-1-deficient strain ( $\triangle E c c C-L u x B C A D E)$ in BMMs.

$(H)$ Luminescent growth assay. BMMs were treated with DNA (Lipo+DNA), transfection reagent alone (Lipo), or IFN- $\beta$ (250U) as indicated. Mean \pm SEM of four replicate samples are shown.

IFN- $\beta$ was added $4 \mathrm{~h}$ pre-infection and DNA was delivered $1 \mathrm{~h}$ post-infection. ${ }^{*} p=0.003$ by t-test (I) BMMs were infected with IpqN mutant Mtb for $6 \mathrm{~h}$ and analyzed by Western blot. 


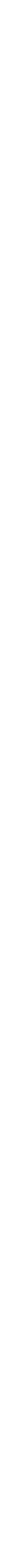

Figure 5 
Figure 6. Model of balance between anti-viral and anti-bacterial cell-intrinsic immune response pathways.

Host macrophages tailor responses to distinct kinds of pathogens at the earliest stages of infection by activating cell-intrinsic immune pathways tailored to the threat, e.g. virus or bacterium. These programs appear to be mutually antagonistic, as activation of antiviral pathways comes at the cost of antibacterial immunity during Mtb infection. Our work indicates that CBL functions to influence this balance by inhibiting viral responses and promoting antibacterial immunity. Macrophage plasticity can be manipulated by pathogens (dashed arrows) to tip the balance to promote inappropriate responses to favor replication. 


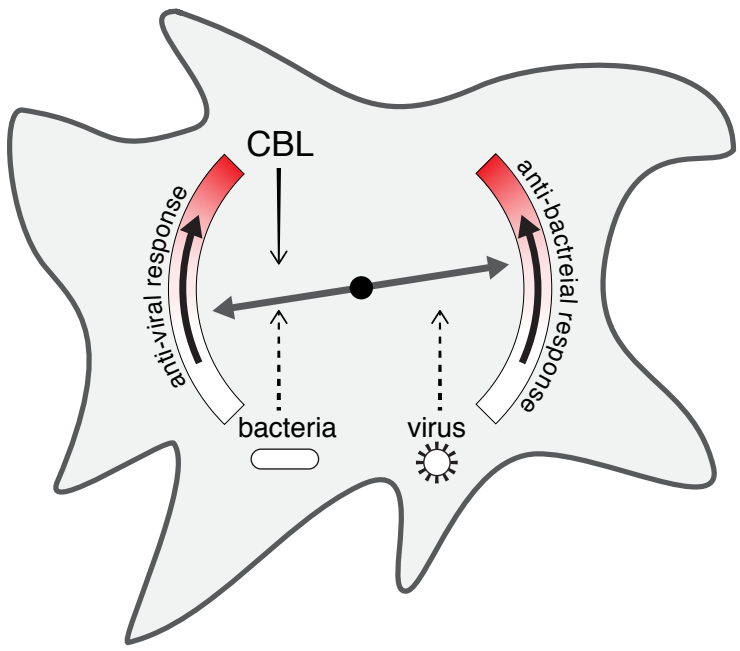

Figure 6 


\section{SUPPLEMENTAL INFORMATION}

Figure S1. Related to Figure 1. Establishing conditions for proteomic analysis of secreted Mtb proteins in human cells.

(A) Culture filtrates were prepared from wild-type cultures, separated by SDS-PAGE, and proteins identified by mass spectrometry. The gel was cut into sequential regions as indicated, and each slice was individually subjected to in-gel trypsin digestion and LC-MS/MS (Table S1). Supernatants from ESX-1 mutants $(\triangle m y c P 1)$ indicate that most of the secreted proteins are not ESX-1 substrates, and cell lysates were added as controls for lysis.

(B) Immunofluorescence microscopy of 293T cells expressing either the full-length uncleaved Mtb Rv1419 protein, or the form predicted to be released after signal peptidase cleavage.

(C) Western blot analysis of U937 macrophages and 293T cells transfected with plasmids encoding the indicated Strep-tagged proteins, showing much higher levels of expression in 293T cells. The majority of proteins were undetectable from U937 cells, but were expressed to sufficient levels in 293T cells to purify and detect host interactors.

(D) Affinity purifications from lysates derived as described in Figure 1 were separated by SDSPAGE and visualized by silver staining, demonstrating the efficient expression and purification of bacterial proteins. Arrowheads denote the tagged bacterial proteins.

(E) Example of reciprocal co-immunoprecipitation validation studies. 293T cells were cotransfected with plasmids expressing Strep-tagged Mtb Rv2074 and with FLAG-tagged versions of GFP (negative control), or each of three putative interactors identified in the PPI map, VPRBP, ATG4B, and VCP. The host proteins were immunoprecipitated with anti-FLAG antibody and Rv2074 was detected by Western blot using anti-Strep antibodies.

\section{Figure S2. Related to Figure 4. The LpqN mutant competes normally in mixed cultures grown in liquid media.}

An aliquot of the pool of eight strains used to infect mice for the in vivo competition assay (Figure 4) was grown in liquid 7H9 media for 10 days and the relative proportion of each strain was quantified by qPCR of the genomic sequence tag. Mean \pm SEM is displayed.

\section{Figure S3. Related to Figure 4. Genetic interactions between Host $\mathrm{Cb}$ and $M$ tb IpqN in RAW264.7 cells.}

(A) The gene encoding VPS33b, a putative LpqN interactor, was mutated by CRISPR/Cas9 in RAW264.7 cells. These cells were infected with the Mtb IpqN mutant strain expressing the IUXBCADE operon from Vibrio harveyi, and bacterial growth was assessed by measuring luminescence. The assay was performed twice with three independent mutant clones and two independent wild-type control clones. Each condition was measured in quadruplicate; mean \pm SEM from representative clones are displayed.

(B) CRISPR/Cas9 homozygous frameshift mutation in Cb/ was created in RAW264.7 and tested with the IpqN IuxBCADE strain as described for (A). The assay was performed three times with four independent mutant clones and two independent wild-type control clones. Each condition was measured in quadruplicate; mean \pm SEM from representative clones are displayed.

(C) $\mathrm{CbI}^{{ }^{-}}$and $\mathrm{CbI}^{+/+}$primary BMMs were infected with the IpqN, luxBCADE mutant and bacterial growth was enumerated by plating for CFU 12 days post-infection.

(D) Growth of the IpqN mutant was also assessed in the $\mathrm{Cbl}^{-/-}$and $\mathrm{CbI}^{+/+}$primary BMMs as 
described in (C) by measuring luminescence. Mean \pm SEM are displayed.

(E) The Cb/ CRISPR/Cas9 clones of RAW264.7 described in (B) were infected with $L$. monocytogenes and CFU enumerated at the indicated times. Mean change in CFU relative to $\mathrm{t}=0$ is shown \pm SEM. Representative results from two independent experiments are shown.

(F) Infection as in (E) with $S$. Typhimurium.

Figure S4. Related to Figure 4. Direct interaction between LpqN and CBL in vitro.

LpqN-Strep was expressed in E. coli BL21DE3 cells, and whole cell lysates were mixed with similar lysates from cells expressing either CBL-FLAG or GFP-FLAG for $3 \mathrm{~h}$ at $4^{\circ} \mathrm{C}$ to allow for binding. LpqN-Strep was affinity purified using Strep-Tactin resin, and co-purification of the host fusion proteins were detected by western blotting using anti-FLAG antibodies.

Table S1. Related to Figure 1. High-confidence set of Mtb secreted proteins.

Mtb culture filtrates separated by SDS-PAGE (Figure S1A) were subjected to LC-MS/MS analysis to identify secreted proteins. These results were manually curated to remove known cytoplasmic or cell-wall contaminants (Målen et al., 2007).

Table S2. Related to Figure 1. Comparison of U937 and 293T co-purifying proteins.

To evaluate the impact of addition of U937 macrophage cell lysates to our PPI scheme (Figure 1A), a subset of bacterial proteins were re-analyzed by AP-MS using 293T lysate alone without inclusion of the U937 lysate. All these interactions were scored by MiST and those with values $\geq 0.7$ are displayed.

Table S3. Related to Figure 1. Full AP-MS dataset.

All host proteins identified from the AP-MS proteomics pipeline described in this study (Figure 1).

Table S4. Related to Figure 1. MiST scoring of AP-MS dataset.

Table S5. Related to Figure 2. Comparison of AP-MS datasets from different pathogens.

Table S6. Related to Figure 2. Functional annotation of high-confidence interacting host proteins.

DAVID v6.8 Uniprot keywords analysis was used to annotate all host proteins with MiST $\geq 0.7$ for the indicated pathogens.

Table S7. Related to Figure 2. Evolutionary analysis of $\boldsymbol{M t b}$-interacting proteins.

SnIPRE and iHS analysis data. 
bioRxiv preprint doi: https://doi.org/10.1101/202598; this version posted October 17,2017 . The copyright holder for this preprint (which was not certified by peer review) is the author/funder. All rights reserved. No reuse allowed without permission.

Table S8. Related to Figure 4. PTMs of host proteins during infection with Mtb. 
bioRxiv preprint doi: https://doi.org/10.1101/202598; this version posted October 17, 2017. The copyright holder for this preprint (which was not

A. certified by peer review) is the author/funder. All rights reserved. No reuse allowed without permission.
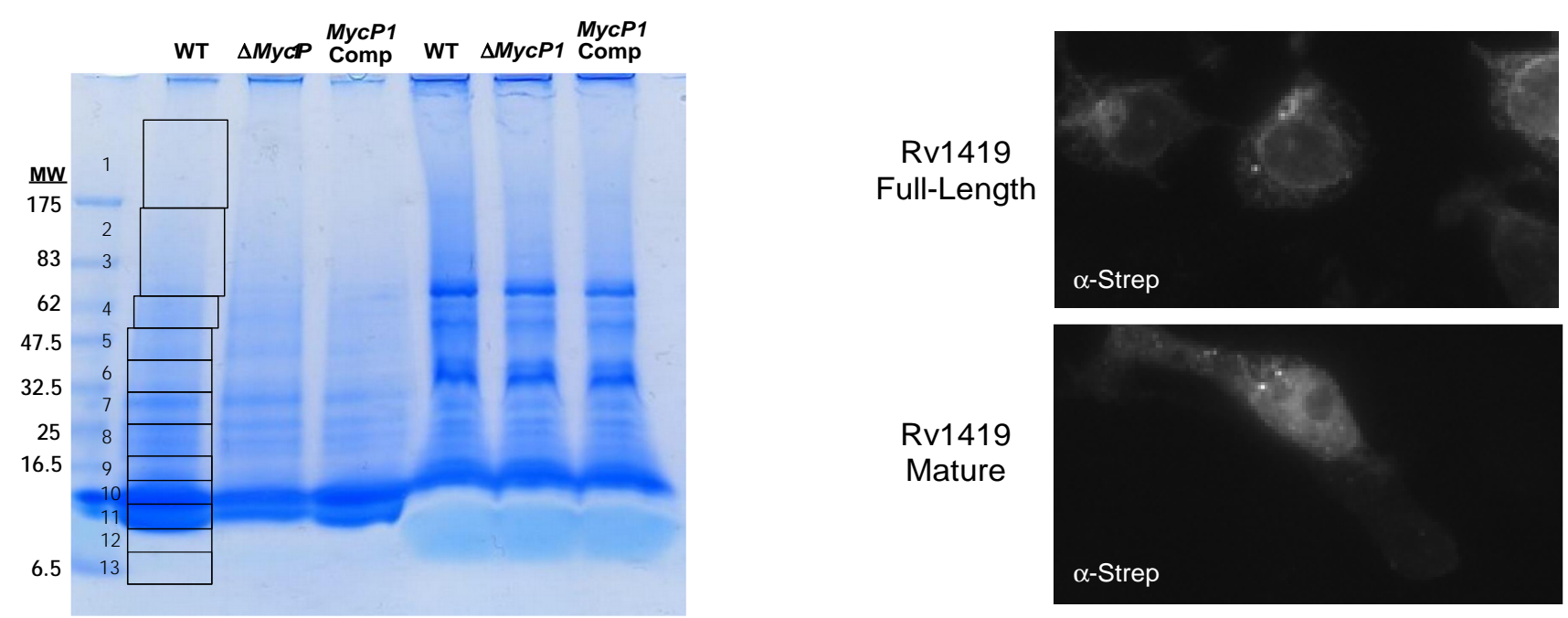

C.

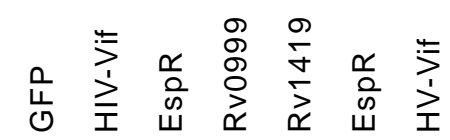

D.

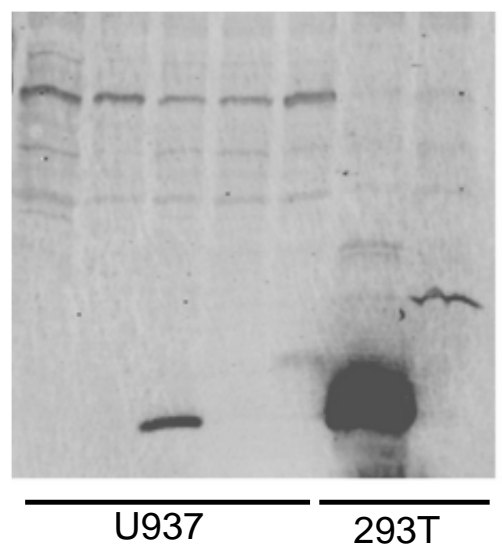

Strep WB
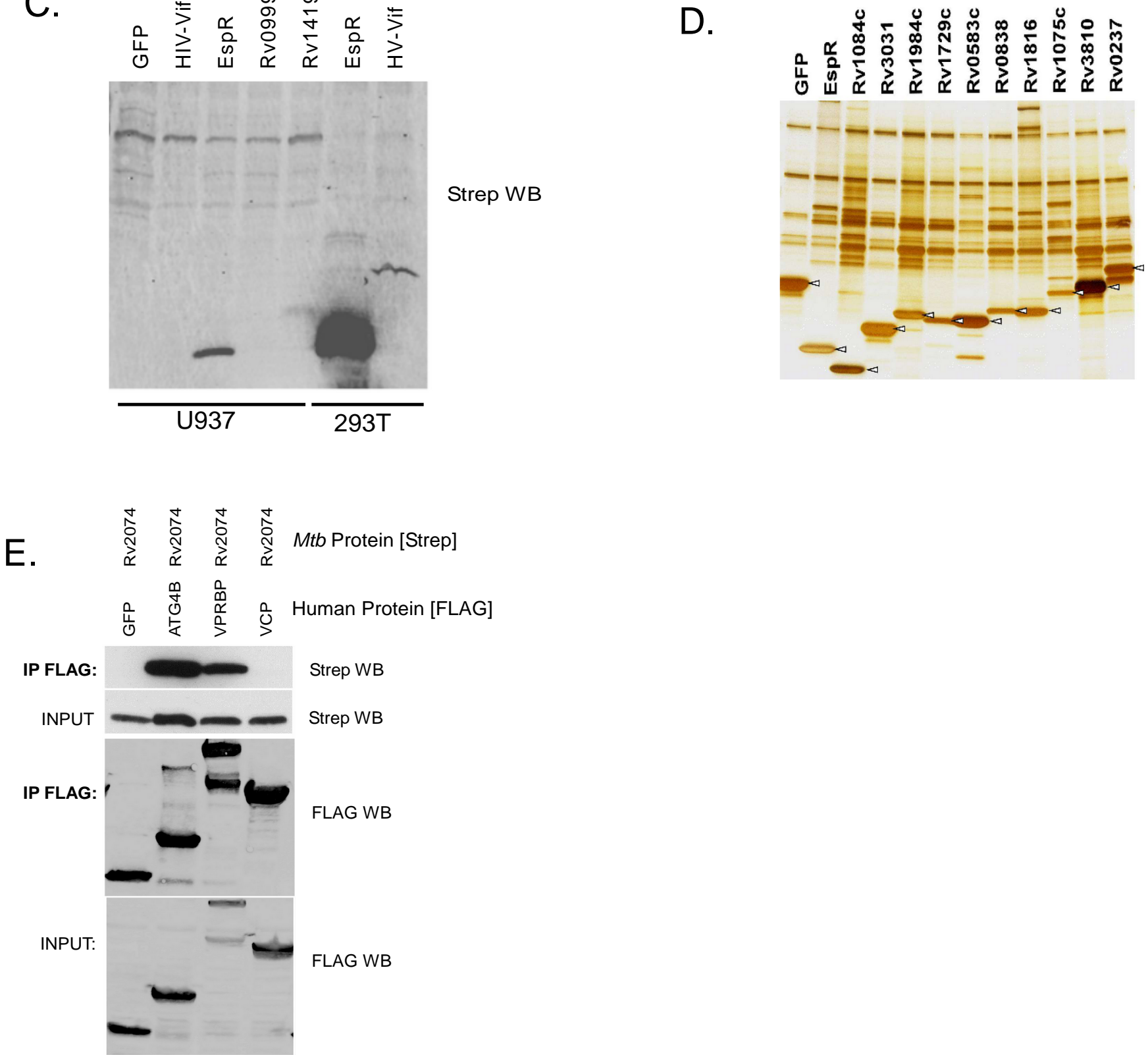
bioRxiv preprint doi: https://doi.org/10.1101/202598; this version posted October 17,2017 . The copyright holder for this preprint (which was not certified by peer review) is the author/funder. All rights reserved. No reuse allowed without permission.

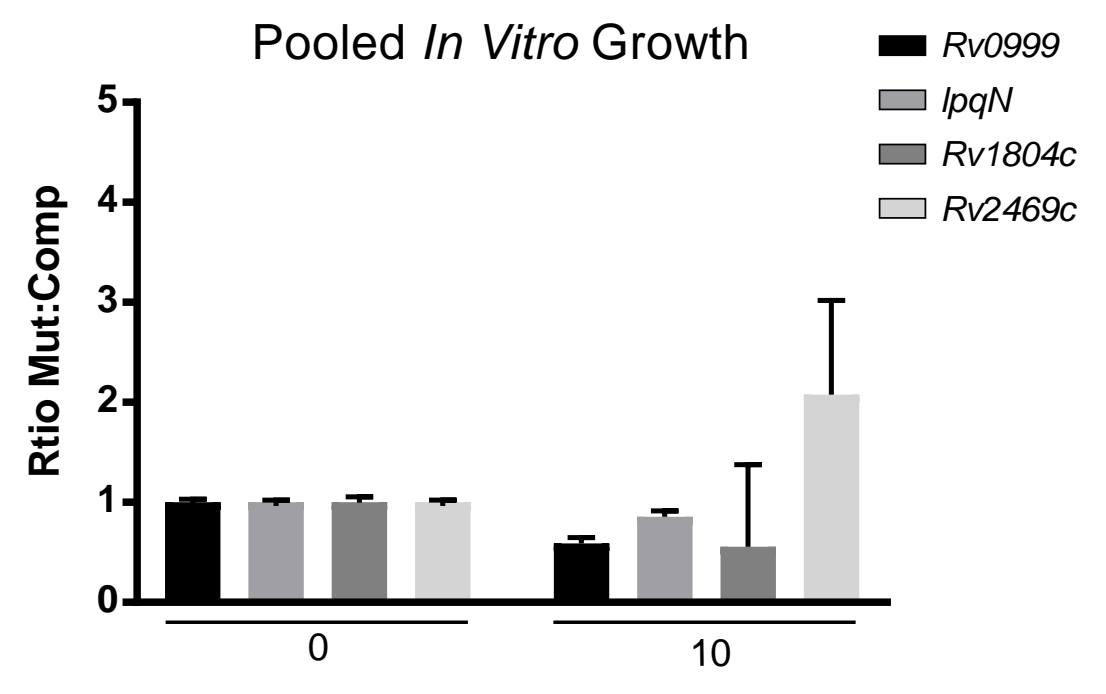

Figure S2 
A.

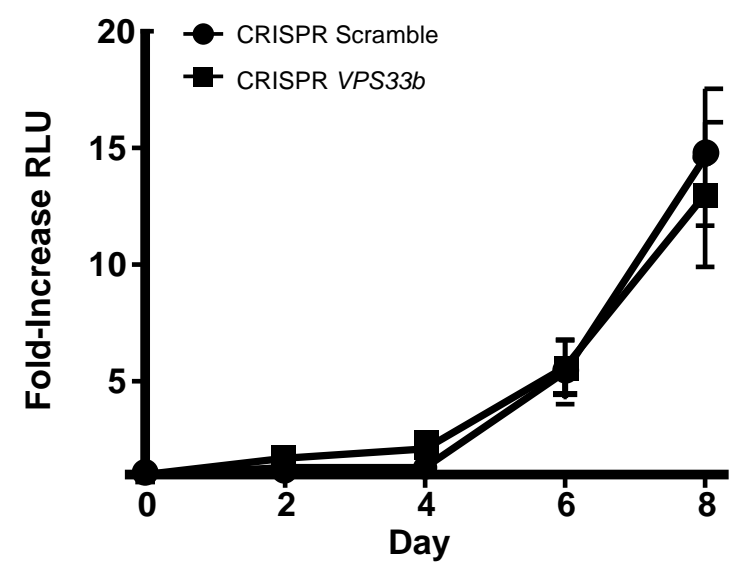

C.

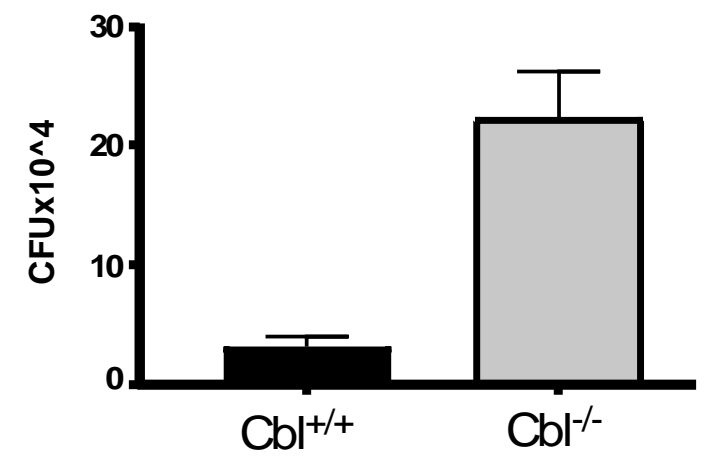

E.

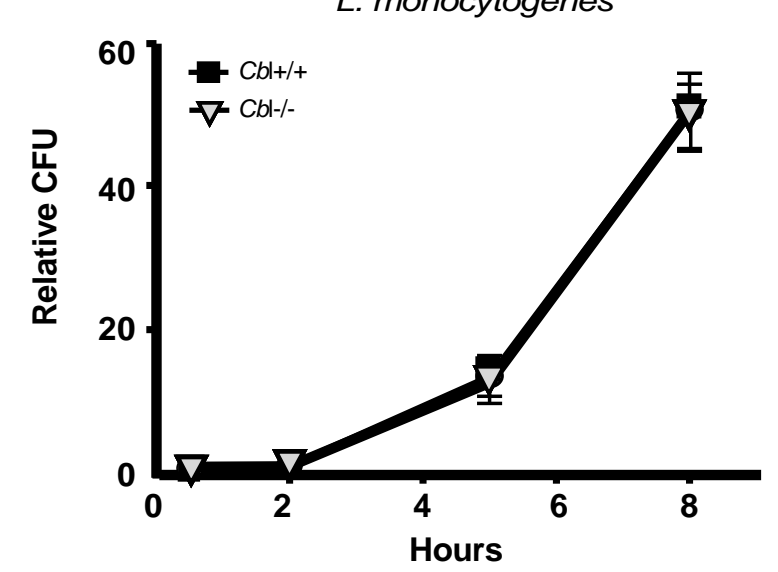

B.

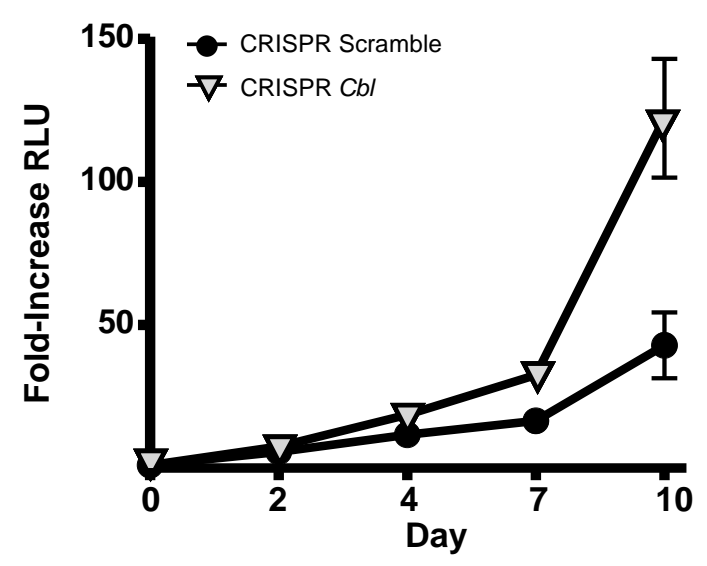

D.

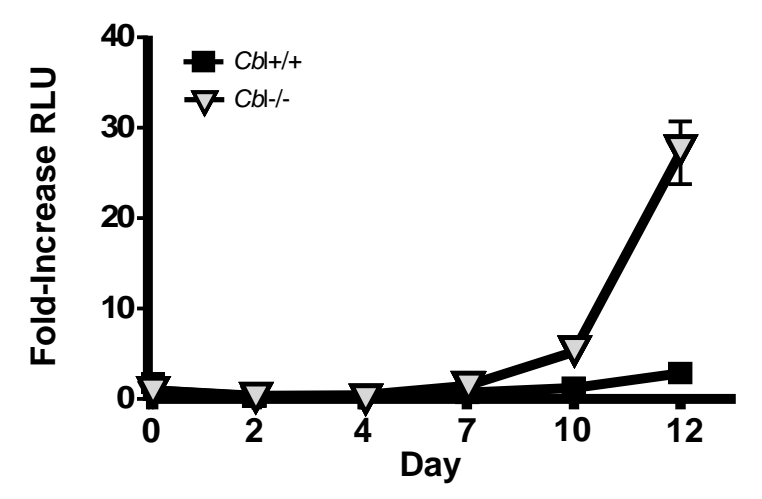

F.

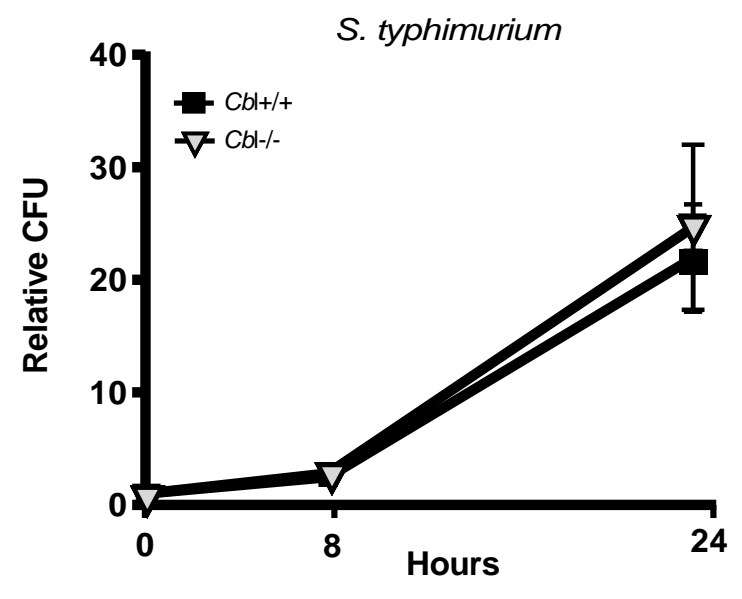




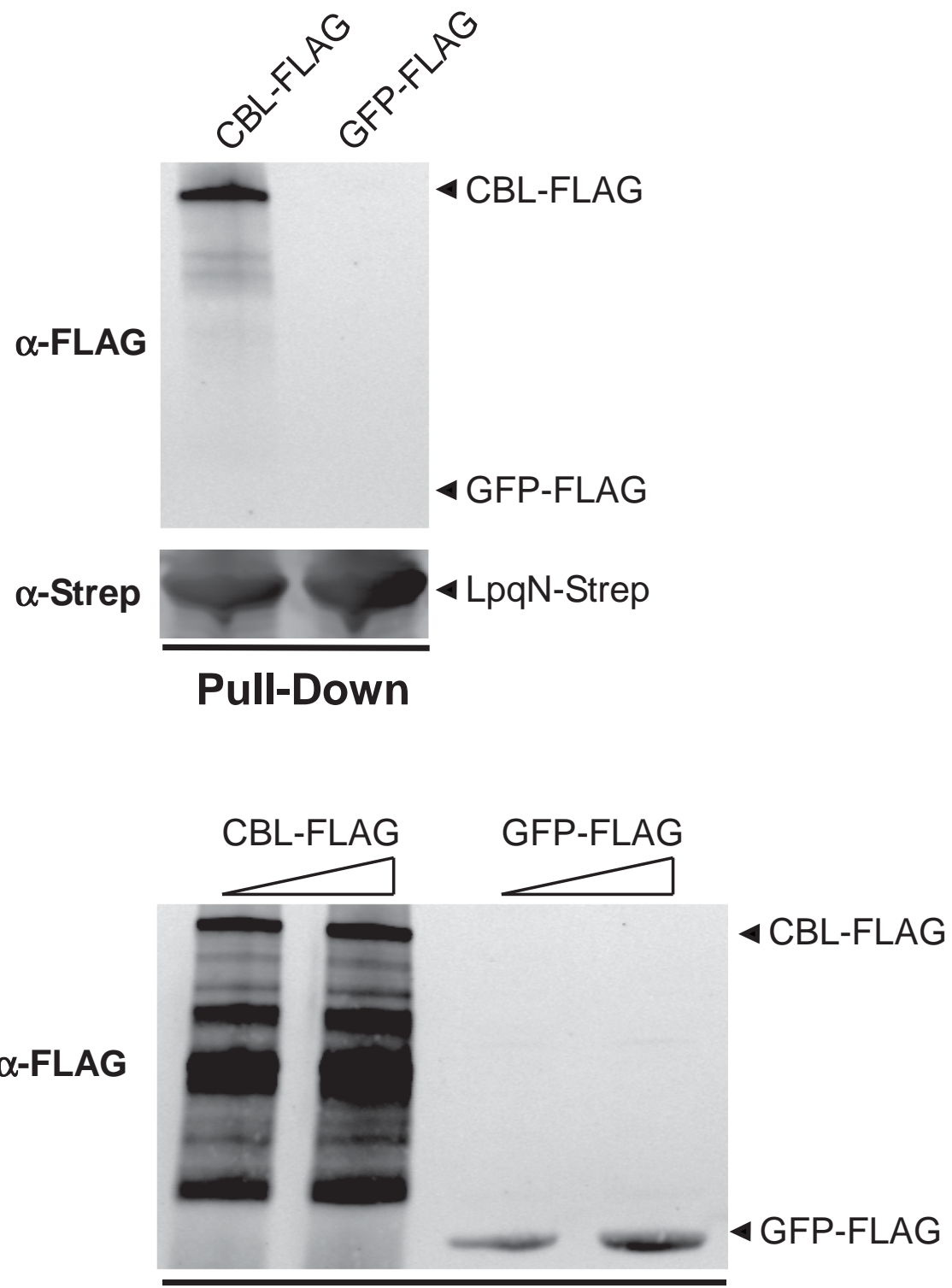

\section{Input}




\section{STAR METHODS}

\section{KEY RESOURCE TABLE}

\section{Bacterial Strains}

Strain

Rv2469 Mutant

Rv1084c Mutant

Rv0999 Mutant

IpqN Mutant

Rv2469 Complement

Rv1084c Complement

Rv0999 Complement

IpqN Complement

ESX-1 Mutant

\section{Antibodies}

Protein

CBL

IRF-3 (D83B9)

P-IRF-3 (S396)

ACTB

UBC

ATP6V0A1

\section{CRISPR Guides}

Cbl Exon 1 Guide 1

Cbl Exon 1 Guide 2

Vps33b Exon 1 Guide 1

Vps33b Exon 1 Guide 2

\section{Genotyping Primers}

Cbl Genotyping Fw

Cb/ Genotyping Rv

Vps33b Genotyping Fw

Vps33b Genotyping Rv
Background

CDC1551

CDC1551

CDC1551

CDC1551

CDC1551

CDC1551

CDC1551

CDC1551

Erdman

Supplier

Santa Cruz Bio.

Cell Signaling

Cell Signaling

Cell Signaling

Cell Signaling

Santa Cruz Bio.

gtgaagaagagctcgggcgc cgggagcgggcggcctgatc ggtcattcatccgtcgagta gccgagtgtgcggatgtcgt

tctcactccetgggctgc cctgcacggtggacaagaaga gatccagagccoaggcaagcg gccacggcaggcactgac
Genotype

Rv2469::Tnhimar1, attB ${ }^{M S 6}$ ::pBUGFP, attB $^{T n 5}$ ::pmv306Lux

Rv1084c::Tnhimar1, attB ${ }^{M S 6}::$ pBUGFP, attB $^{T n 5}::$ pmv306Lux

Rv0999::Tnhimar1, attB ${ }^{M S 6}:$ :pBUGFP, attB $^{T n 5}::$ pmv306Lux

IpqN::Tnhimar1, attB ${ }^{M S 6}::$ BBUGFP, attB ${ }^{T n 5}$ ::pmv306Lux

Rv2469::Tnhimar1, attB ${ }^{M S 6}:$ :pBURv2469, attB $^{T n 5}:$ :pmv306Lux

Rv1084c::Tnhimar1, attB ${ }^{M S 6}:: \mathrm{pBU} R v 1084 \mathrm{c}$, attB $^{T n 5}::$ pmv306Lux

Rv0999c::Tnhimar1, attB ${ }^{M S 6}:$ :pBURv0999, attB $^{T n 5}:$ :pmv306Lux

IpqN::Tnhimar1, attB ${ }^{M S 6}:: p B U / p q N$, attB $^{\text {Tn5 }}$ ::pmv306Lux

$\Delta \operatorname{eccCa}_{1}-\Delta e c c C b_{1}, \operatorname{attB}^{T n 5}::$ pmv306Lux

\section{Catalog \#}

sc-1651

$4302 S$

$4947 S$

$8457 \mathrm{~T}$

FK2

sc-28801

\section{RT-qPCR}

Gene 


\begin{tabular}{|c|c|}
\hline Actin(Fw) & ggtgtgatggtgggaatgg \\
\hline Actin(Rv) & gccctcgtcacccacatagga \\
\hline IFN- $\beta(F w)$ & tccgagcagagatcttcaggaa \\
\hline IFN- $\beta(R v)$ & tgcaaccaccactcattctgag \\
\hline Ifit1(Fw) & cgtagcctatcgccaagattta \\
\hline Ifit1Rv(Rv) & agctttagggcaaggagaac \\
\hline \multicolumn{2}{|l|}{$\begin{array}{l}\text { In Vivo Competition } \\
\text { Assay Primers }\end{array}$} \\
\hline Gene/Tag & Sequence \\
\hline \multicolumn{2}{|l|}{ Rv0999 } \\
\hline Tb50comp_Qtag(Fw) & atcgatgtcgacgtagttccg \\
\hline Tb50comp_Qtag(Rv) & caggttgtgacaggatcggac \\
\hline Qtag_5Fw) & aagcttatcgatgtcgacgtagtttc \\
\hline Qtag_5(Rv) & gcagtcgatcgtacgctagttagc \\
\hline \multicolumn{2}{|l|}{$\operatorname{LpqN}(R v 0583 c)$} \\
\hline 60cQtag(Fw) & ccgcggtaccagatctttagc \\
\hline 60cQtag(Rv) & cgcgaccctatcactgcaatc \\
\hline Qtag_2(Fw) & acgtaaacggccacaagttcagc \\
\hline Qtag_2(Rv) & cttcagggtcagcttgccgtag \\
\hline \multicolumn{2}{|l|}{ Rv1804c } \\
\hline 70cQtag(Fw) & agggaacaaaagctggagcc \\
\hline 70cQtag(Rv) & gcgcggatataggccactg \\
\hline Qtag_4(Fw) & ccgaccacatgaagcagcacg \\
\hline Qtag_4(Rv) & tggtgcgctcctggacgtag \\
\hline \multicolumn{2}{|l|}{$R v 2469 c$} \\
\hline 84cQtag(Fw) & ccgcggtaccagatcttttgatc \\
\hline 84cQtag(Rv) & cacgccaaatgccacgtc \\
\hline Pbu_QTag5 (Fw) & cgtcgtccttgaagaagaccag \\
\hline Pbu_QTag5(Rv) & acacaggaaacagctatgaccttg \\
\hline
\end{tabular}

\section{CONTACT FOR REAGENT AND RESOURCE SHARING}

Further information and requests for resources and reagents should be directed to and will be fulfilled by the Lead Contact, Jeffery Cox (jeff.cox@berkeley.edu).

\section{EXPERIMENTAL MODEL AND SUBJECT DETAILS}

\section{Macrophages}

Bone marrow-derived macrophages (BMMs) were isolated by flushing the femurs from 8-10-week-old B6 female mice. They were cultured in high-glucose DMEM supplemented with $20 \%$ heat-inactivated FCS and $10 \%$ conditioned media from 3T3MCSF cells. All cells were cultured with at $37^{\circ} \mathrm{C}$ with $5 \% \mathrm{CO}_{2} . \mathrm{CbI}^{+/+}$and $\mathrm{CbI}^{/-} \mathrm{BMMs}$ were isolated from mice of the following genotypes: 


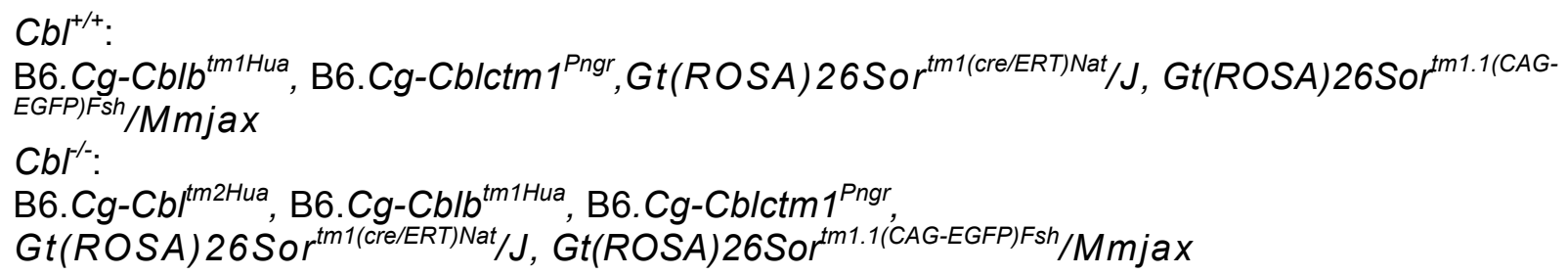

Both $\mathrm{Cbl}^{+/+}$and $\mathrm{Cbl}^{--}$BMMs were then cultured in the presence 1uM 4-hydroxytamoxifen (Sigma) to trigger recombination of the floxed Cbl allele (Mukhopadhyay et al., 2016).

\section{Cell Lines}

RAW 264.7 mouse macrophages and 293T cells were purchased from ATCC and cultured according to ATCC recommendations with addition of 20mM HEPES pH7.4. 293T were purchased from ATCC and cultured according to ATCC recommendations.

\section{Bacterial strains}

All Mtb strains were cultured in $7 \mathrm{H} 9$ liquid media (BD) supplemented with $10 \%$ Middlebrook OADC (Sigma), $0.5 \%$ glycerol, $0.05 \%$ Tween 80 in roller bottles at $37^{\circ} \mathrm{C}$. Transposon mutants were obtained from ATCC and carry Himar1 transposon insertions with the Kanamycin resistance gene in the indicated locus. Transposon insertion sites were validated by PCR and Sanger sequencing (Lamichhane et al., 2003). For genetic complementation studies, the predicted promoter region and open reading frame were cloned into the Sacl site of the integrating vector pBU (Vultos et al., 2006) which had been modified to confer Zeocin resistance, and strains were selected with $25 \mathrm{ug} / \mathrm{ml}$ Zeocin. Control strains carried pBU with a $\sim 100$ nucleotide fragment of GFP as unique sequence tag. For luminescent growth assays, strains carried the containing a codon-optimized luxBCADE operon expressed from a MOPS promoter carried on the pmv306-Hyg integrating vector (Craney et al., 2007). The $\Delta$ eccCa ${ }_{1}$ $\triangle e c c C b_{1}$ strain was made by specialized transduction using the pMSG361 vector (Rosenberg et al., 2015).

\section{METHOD DETAILS}

\section{Mtb secreted protein analysis}

Mtb Erdman strain was grown in 7H9+OADC (BD Biosciences), +0.05\% Tween-80 (Sigma) until mid-log, then transferred to Sauton's minimal media $+0.05 \%$ Tween- 80 for $5 \mathrm{~d}$, and then finally to Sauton's media with $0.005 \%$ Tween for $5 \mathrm{~d}$. Bacteria were pelleted and supernatants concentrated with a $3 \mathrm{~K}$ MWCO filter (Millipore). Bacteria were lysed by boiling, followed by 10 minutes of sonication in $1 \%$ SDS. 20 ug each of cell lysate and culture filtrate were separated by SDS-PAGE and western blot performed against GroEL to verify that there was no detectable contamination of the culture filtrate by cytoplasmic proteins. For MS analysis, protein was resolved on SDS-PAGE, visualized by Coomassie, and gel slices subjected to trypsin digestion as described below.

\section{In vivo competition assay}

12-week-old female B6 mice were inoculated by aerosol with a pool of four mutant strains and cognate complemented strains delivered at $\sim 75 \mathrm{CFU}$ of each strain using a Madison chamber device. At the indicated times, lungs of 4-5 mice were homogenized and plated on $7 \mathrm{H} 10$ plates. $\sim 10,000$ individual colonies were scraped into Trizol (Thermo Fisher Scientific). Samples were then lysed using silica beads and bead-beating and RNA removed from Trizol per manufacturer instructions for RNA isolation. Genomic DNA was then isolated from organic phase by backextraction of with $4 \mathrm{M}$ guanidine thiocyanate/50mM Sodium Citrate/1M Tris base, and the 
aqueous phase containing DNA was removed. The $\mathrm{pH}$ was normalized by adding 0.2 volumes sodium acetate, and 0.4 volumes ethanol was added. DNA was then purified over RNeasy columns (Quiagen) according to manufacturer instructions. qPCR primers were designed to detect unique sequence tags inserted in each strain's genome using either a fragment of the GFP gene (mutant strains) or the unique junction between the pBU vector \& complementation cassette (complemented strains). Each primer set was verified to detect only its specific strain. qPCR was conducted with Taq polymerase (NEB) and SYBR green I (Sigma) detection.

\section{Ex vivo luminescent bacterial growth assay}

$M t b$ strains carrying the luxBCADE operon were prepared for inoculation by washing twice in PBS, removing aggregates with a 200 RCF spin, followed by gentle sonication to generate a fine bacterial suspension. Bacteria were opsonized in 10\% heat-inactivated horse serum and macrophages infected at an MOI=2 by spinning inoculated plates for 10 min at $400 \mathrm{RCF}$. Monolayers were washed, and bacterial luminescence measured the indicated times using a Spectromax L (Molecular Dynamics). Macrophage growth media was changed daily for cell lines and every 48h for BMM cultures.

\section{CRISPR mutagenesis}

CRISPR guides were designed to target regions in the first exon of target genes using an online bioinformatic tool (http://crispr.mit.edu), and cloned into the BsmB1 site of the pXPR_001 vector (a gift from Feng Zhang (Addgene plasmid \# 52961)). Viral particles were produced in Lenti-X cells (Clontech) per manufacturer's instructions, and used to transduce RAW264.7 cells. Therese were selected in 5ug/ml Puromycin (InVivoGen), and single-cell clones isolated. Mutations were identified by using PCR to amplify the first exon by PCR followed by Sanger sequencing. CRISPR CBL mutants were independently confirmed by western blot with a mouse anti-c-CBL antibody (Santa Cruz Biotechnology cat\# sc-1651).

\section{Western blots}

Protein in lysates was quantified by BCA (Pierce Fisher Scientific). 20ug of cell lysate was separated by SDS-PAGE (Bio-Rad TGX), and transferred onto nitrocellulose membranes. After probing with the indicated antibodies, membranes were then imaged on an Odyssey scanner (Li-cor).

\section{Affinity purification}

Mtb genes were amplified by PCR from Erdman strain genomic DNA. For genes with a predicted signal peptide using SignalP 3.0 (Bendtsen et al., 2004), the portion of the open reading frame corresponding to the mature protein was amplified. Genes were cloned into PCDNA4 with a C-terminal 2x-Strep tag and expressed in 293T cells using calcium phosphate transfection. Cells were lysed $36 \mathrm{~h}$ later in IP buffer (50mM Tris 7.4, $150 \mathrm{mM} \mathrm{NaCl}, 1 \mathrm{mM}$ EDTA, $0.05 \%$ NP-40) with phosphatase and protease inhibitors (Roche) and tagged proteins immobilized on Strep-Tactin resin (IBA). Macrophage lysate was generated from U937 cells differentiated with 10nM PMA for $72 \mathrm{~h}$ and then similarly lysed in IP buffer. $10 \mathrm{mg}$ of macrophage lysate was added to each immobilized bacterial factor and binding allowed to proceed overnight. The resin was then washed $4 x$ in IP buffer, and twice in IP buffer without NP-40 before elution in $10 \mathrm{mM}$ biotin. For reciprocal immunoprecipitation using FLAG-tagged human proteins the human factors were cloned as 3xFLAG-tag fusion proteins in pCDNA4. Combinations of host and bacterial factors were co-transfected into 293T cells using calcium phosphate and immunoprecipitation with anti-FLAG M2 antibody was performed using the same lysis and wash conditions as above.

\section{Mass Spectrometry}

Purified proteins eluates were digested with trypsin for LC-MS/MS analysis. Samples were 
denatured and reduced in $2 \mathrm{M}$ urea, $10 \mathrm{mM} \mathrm{NH} 4 \mathrm{HCO} 3,2 \mathrm{mM}$ DTT for $30 \mathrm{~min}$ at $60 \mathrm{C}$, then alkylated with $2 \mathrm{mM}$ iodoacetamide for $45 \mathrm{~min}$ at room temperature. Trypsin (Promega) was added at a 1:100 enzyme:substrate ratio and digested overnight at 37C. Following digestion, samples were concentrated using C18 ZipTips (Millipore) according to the manufacturer's specifications. Desalted samples were evaporated to dryness and resuspended in $0.1 \%$ formic acid for mass spectrometry analysis.

For the MS study to establish a high-confidence set of Mtb secreted proteins culture filtrates were resolved by SDS-PAGE. The gel was partitioned into sequential slices and each of these was individually subjected to in-gel trypsin digestion followed by desalting and LC-MS/MS analysis on a Thermo Scientific LTQ XL linear ion trap mass spectrometer. The LTQ XL system was equipped with a LC Packings Ultimate HPLC with an analytical column (10 cm x $75 \mathrm{um}$ I.D. packed with ReproSil Pur C18 AQ 5 um particles). The system delivered a gradient from 5\% to $30 \% \mathrm{ACN}$ in $0.1 \%$ formic acid over one hour, and collected data in a data-dependent fashion. The LTQ XL collected one full scan followed by 10 collision-induced dissociation MS/MS scans of the 10 most intense peaks from the full scan. Dynamic exclusion was enabled for 30 seconds with a repeat count of 1 .

For the primary AP-MS study used to establish the interactome, digested peptide mixtures were analyzed by LC-MS/MS on a Thermo Scientific LTQ XL linear ion trap mass spectrometer as above.

For the secondary analysis, where AP-MS was performed without inclusion of U937 macrophage lysate, the digested peptide mixtures were analyzed by LC-MS/MS on a Thermo Scientific Velos Pro dual linear ion trap 238 mass spectrometer equipped with an Easy-nLC II HPLC with a pre-column (2 cm x 100 um I.D. packed with 239 ReproSil Pur C18 AQ 5 um particles) and an analytical column (10 cm x 75 um I.D. packed with ReproSil Pur 240C18 AQ 3 um particles). A gradient was delivered from $5 \%$ to $30 \% \mathrm{ACN}$ in $0.1 \%$ formic acid over one hour. 241. The mass spectrometer collected data in a data-dependent fashion with one full scan followed by 20 collision-242 induced dissociation MS/MS scans of the 20 most intense peaks from the full scan. Dynamic exclusion was 243 enabled for 30 seconds with a repeat count of 1 .

The results raw data was matched to protein sequences by the Protein Prospector algorithm (Clauser et al., 1999). Data were searched against a database containing Swiss Prot Human protein sequences (downloaded March 6, 2012), and concatenated to a decoy database where each sequence was randomized in order to estimate the false positive rate. The searches considered a precursor mass tolerance of $1 \mathrm{Da}$ and fragment ion tolerances of $0.8 \mathrm{Da}$, and considered variable modifications for protein $\mathrm{N}$-terminal acetylation, protein $\mathrm{N}$-terminal acetylation and oxidation, glutamine to pyroglutamate conversion for peptide $\mathrm{N}$-terminal glutamine residues, protein $\mathrm{N}$-terminal methionine loss, protein $\mathrm{N}$-terminal acetylation and methionine loss, and methionine oxidation, and constant modification for carbamidomethyl cysteine. Prospector data was filtered using a maximum protein expectation value of 0.01 and a maximum peptide expectation value of 0.05 .

Our global identification of phosphorylation and ubiquitylation during Mtb infection of RAW264.7 macrophages is described in a separate manuscript (Parry et. al., in preparation) and will be submitted to ProteomeXchange via PRIDE.

\section{MiST}

AP-MS samples were scored with Mass spectrometry Interaction STatistics (MiST) algorithm, using the MiST reproducibility (0.45), specificity $(0.50)$ and abundance $(0.05)$ weights previously published (Davis et al., 2015). All bait-prey pairs with a MIST score $\geq 0.70$ were considered confident interactions and were combined with human protein interactions from the CORUM and STRING databases. The resulting network diagram was plotted using Cytoscape, v.3.1.2 411 (Smoot et al., 2011). For the subset re-analysis without U937 lysate, MiST with identical settings 
was used but additional control Strep-tag purifications added to the analysis matrix in MiST to compensate for the smaller number of experimental samples and establish robust specificity scoring.

\section{Evolutionary Analysis}

For analysis over a 5-7 million-year timescale we employed SnIPRE (Eilertson et al., 2012) which is a Bayesian mixed effects model of the McDonald-Kreitman test (McDonald and Kreitman, 1991). We focus our analysis on the human lineage by removing sites that are inferred to have received mutations along the chimpanzee lineage based on an alignment of closely related primates. For analysis of more recent evolution in the primate lineage we used a modified version of integrated Haplotype Score (iHS) (Voight et al., 2006) as implemented in (Szpiech and Hernandez, 2014).

\section{In Vitro Interaction Studies}

Proteins were cloned into the $\mathrm{pH} 3 \mathrm{C}$ vector as either a Strep-Tag fusion (LpqN) or FLAG-Tag fusions (CBL, GFP). Factors were individually expressed in BL21 cells by overnight induction with IPTG at room-temperature. Cells were lysed in PBS + protease inhibitors using lysozyme and sonication. Total cell lysates containing $\sim 10 \mathrm{ug}$ of each tagged protein were combined, subjected to Strep-Tactin purification, and interacting proteins were detected by western blotting as above.

\section{Gene Expression Analysis}

Infected macrophages were lysed in Trizol (Thermo Fisher Scientific) and then purified with silica spin-columns (Purelink, Ambion) per manufacturer instructions. cDNA was generated using 500ng total RNA with the Superscript III First Strand Synthesis Kit (Invitrogen), and subjected to qPCR as described above with normalization to the beta-actin transcript.

\section{Immunofluorescence microscopy}

Coverslips were fixed for $20 \mathrm{~min}$ in $4 \%$ PFA, permeabilized in $0.05 \%$ Saponin (Sigma) and incubated with the indicated antibodies for $3 \mathrm{~h}$ at RT. Mycobacteria were visualized either by expression of mCherry or by labeling with SytoBC (Fisher). All samples were mounted and images acquired on a Keyence BZ-X700 microscope. Z-stacks at $0.5 \mathrm{uM}$ were acquired and maximum intensity projections generated. To quantify colocalization positive phagosomes were scored for each marker by a microscopist blinded to sample identity.

\section{QUANTIFICATION AND STATISTICAL ANALYSIS}

\section{Statistical Analysis}

For RT-qPCR, each sample was amplified in triplicate and transcript levels normalized to the beta-actin gene and the standard error of the mean was calculated. Each experiment was repeated at least two times from separate biological samples with representative data from one biological replicate shown. For luminescent growth assays each sample was quantified in quadruplicate wells and standard-error of the mean was calculated. Each experiment was repeated at least three times from separate biological samples with representative data from one biological replicate shown. For in vivo competition assays the sequence tags were amplified in triplicate qPCR reactions for each mouse, and the ratio between mutant and complement calculated. For the set of mice at each time point, the mean mutant:complement ratio and standard-error of mean were calculated. A Kruskal-Wallis test was used to calculate $p$-values for comparisons among sets of mice. 


\section{Bioinformatic Analysis of Host Proteins}

The set of high-confidence host interacting proteins was analyzed using the DAVID Bioinformatics Resources 6.8 functional annotation tool. Uniprot accession numbers were matched with Uniprot keyword categorization to map protein function and determine enrichment relative to the human proteome. Thresholds for inclusion were set to two-fold enrichment with a Benjamini-Hochberg corrected $p$-value of $<0.05$ to adjust for multiple hypotheses testing. Categories were manually curated to remove redundant and nonspecific categorizations. For overlap analysis between datasets a Fisher's exact test was used with the background proteome defined empirically as the set of all proteins detectable by our Velos Pro dual linear ion trap instrument in 293T cell-related experiments (10,782 proteins)

\section{DATA AND SOFTWARE AVAILABILITY}

The MiST algorithm is available freely on github (https://github.com/kroganlab/mist), and the Comppass algorithm at (https://github.com/RGLab/COMPASS). All MS data will be deposited in the PRIDE database

\section{REFERENCES}

Bendtsen, J.D., Nielsen, H., von Heijne, G., and Brunak, S. (2004). Improved prediction of signal peptides: SignalP 3.0. J Mol Biol 340, 783-795.

Clauser, K.R., Baker, P., and Burlingame, A.L. (1999). Role of accurate mass measurement (+/$10 \mathrm{ppm}$ ) in protein identification strategies employing MS or MS/MS and database searching. Anal Chem 71, 2871-2882.

Craney, A., Hohenauer, T., Xu, Y., Navani, N.K., Li, Y., and Nodwell, J. (2007). A synthetic luxCDABE gene cluster optimized for expression in high-GC bacteria. Nucleic Acids Res 35, e46.

Davis, Z.H., Verschueren, E., Jang, G.M., Kleffman, K., Johnson, J.R., Park, J., Von Dollen, J., Maher, M.C., Johnson, T., Newton, W., et al. (2015). Global mapping of herpesvirus-host protein complexes reveals a transcription strategy for late genes. Mol Cell 57, 349-360.

Eilertson, K.E., Booth, J.G., and Bustamante, C.D. (2012). SnIPRE: selection inference using a Poisson random effects model. PLoS Comput Biol 8, e1002806.

Lamichhane, G., Zignol, M., Blades, N.J., Geiman, D.E., Dougherty, A., Grosset, J., Broman, K.W., and Bishai, W.R. (2003). A postgenomic method for predicting essential genes at subsaturation levels of mutagenesis: application to Mycobacterium tuberculosis. Proc Natl Acad Sci U S A 100, 7213-7218.

McDonald, J.H., and Kreitman, M. (1991). Adaptive protein evolution at the Adh locus in Drosophila. Nature 351, 652-654.

Mukhopadhyay, C., Triplett, A., Bargar, T., Heckman, C., Wagner, K.U., and Naramura, M. (2016). Casitas B-cell lymphoma (Cbl) proteins protect mammary epithelial cells from proteotoxicity of active c-Src accumulation. P Natl Acad Sci USA 113, E8228-E8237.

Rosenberg, O.S., Dovala, D., Li, X., Connolly, L., Bendebury, A., Finer-Moore, J., Holton, J., Cheng, Y., Stroud, R.M., and Cox, J.S. (2015). Substrates Control Multimerization and Activation of the Multi-Domain ATPase Motor of Type VII Secretion. Cell 161, 501-512. Smoot, M.E., Ono, K., Ruscheinski, J., Wang, P.L., and Ideker, T. (2011). Cytoscape 2.8: new features for data integration and network visualization. Bioinformatics 27, 431-432.

Szpiech, Z.A., and Hernandez, R.D. (2014). selscan: an efficient multithreaded program to 
perform EHH-based scans for positive selection. Mol Biol Evol 31, 2824-2827.

Voight, B.F., Kudaravalli, S., Wen, X., and Pritchard, J.K. (2006). A map of recent positive selection in the human genome. PLoS biology 4, e72.

Vultos, T.D., Mederle, I., Abadie, V., Pimentel, M., Moniz-Pereira, J., Gicquel, B., Reyrat, J.M., and Winter, N. (2006). Modification of the mycobacteriophage Ms6 attP core allows the integration of multiple vectors into different tRNAala T-loops in slow- and fast-growing mycobacteria. BMC Mol Biol 7, 47. 